\title{
GLIVENKO-CANTELLI THEORY, ORNSTEIN-WEISS QUASI-TILINGS, AND UNIFORM ERGODIC THEOREMS FOR DISTRIBUTION-VALUED FIELDS OVER AMENABLE GROUPS
}

\author{
By CHRISTOPH SCHUMACHER* ${ }^{*}$ FABIAN SCHWARZENBERGER ${ }^{\dagger}$ AND \\ IVAN VESELIĆ*
}

TU Dortmund* and HTW Dresden ${ }^{\dagger}$

\begin{abstract}
We consider random fields indexed by finite subsets of an amenable discrete group, taking values in the Banach-space of bounded right-continuous functions. The field is assumed to be equivariant, local, coordinate-wise monotone and almost additive, with finite range dependence. Using the theory of quasi-tilings we prove an uniform ergodic theorem, more precisely, that averages along a Følner sequence converge uniformly to a limiting function. Moreover, we give explicit error estimates for the approximation in the sup norm.
\end{abstract}

1. Introduction. Ergodic theorems for Banach space valued functions or fields have been studied among others in $[6,7,11]$ in a combinatorial setting. The three quoted papers consider different group actions in increasing generality: the lattice $\mathbb{Z}^{d}$, monotilable amenable discrete groups and general amenable discrete groups, respectively. Note that amenability is a natural assumption for the validity of the ergodic theorem, as shown explicitly in [14]. Already before that combinatorial ergodic theorems for Banach space valued functions have been proven in the context of Delone dynamical systems; see [8] and the references therein.

The combinatorial framework offers the advantage of a minimum of probabilistic or measure theoretic assumptions, the necessary one being that frequencies or densities of finite patterns are well defined and can be approximated by an exhaustion (corresponding to a law of large numbers). A disadvantage of the combinatorial approach chosen is that the range of colors (or the alphabet corresponding to the values of the random variables) needs to be finite. Also, the derived ergodic theorems are in a sense conditional: The convergence bound depends on the speed of convergence of the pattern frequencies.

Our present research aims at dispensing with the finiteness condition on the set of colors. The price to pay is that we have to assume more probabilistic structure and in particular independence or at least finite range correlations. In return, this structure yields automatically quantitative approximation error bounds. No extra assumptions on the speed of convergence of the pattern frequencies are needed.

Received June 2017.

MSC2010 subject classifications. 60F99, 60B12, 62E20, 60K35.

Key words and phrases. Følner sequence, amenable group, quasi-tilings, Glivenko-Cantelli theory, Uniform convergence, Empirical measures. 
For the case of fields defined over $\mathbb{Z}^{d}$ and $\mathbb{Z}^{d}$-actions, we have established such an ergodic theorem in [12], which takes on the form of a Glivenko-Cantelli theorem, and which we recall now in an informal way.

THEOREM A ([12]). Let $\Lambda_{n}=[0, n)^{d} \cap \mathbb{Z}^{d}$, and $\omega=\left(\omega_{g}\right)_{g \in \mathbb{Z}^{d}} \in \mathbb{R}^{\mathbb{Z}^{d}}$ be an i.i.d. sequence of real random variables. Assume the field

$$
f: \mathcal{P}\left(\mathbb{Z}^{d}\right) \times \mathbb{R}^{\mathbb{Z}^{d}} \rightarrow \mathbb{B}:=\{D: \mathbb{R} \rightarrow \mathbb{R} \mid D \text { right-continuous and bounded }\}
$$

is $\mathbb{Z}^{d}$-equivariant, monotone in each coordinate $\omega_{g}$, local and almost additive, that is, for disjoint $Q_{1}, \ldots, Q_{n} \subseteq \mathbb{Z}^{d}$ and $Q:=\bigcup_{i=1}^{n} Q_{i}$ we have

$$
\left\|f(Q, \omega)-\sum_{i=1}^{n} f\left(Q_{i}, \omega\right)\right\|_{\infty} \leq \sum_{i=1}^{n}\left|\partial Q_{i}\right|,
$$

where $\partial Q_{i}$ denotes the boundary set. Assume furthermore that $f_{\infty}:=\sup _{\omega} \| f(\mathrm{id}$, $\omega) \|_{\infty}<\infty$.

Then there is a function $f^{*}: \mathbb{R} \rightarrow \mathbb{R}$ such that for each $m \in \mathbb{N}$, there exist $a(m), b(m)>0$, such that for all $j \in \mathbb{N}, j>2 m$, there is an event $\Omega_{j, m} \subseteq \mathbb{R}^{\mathbb{Z}^{d}}$, with the properties

$$
\mathbb{P}\left(\Omega_{j, m}\right) \geq 1-b(m) \exp \left(-a(m)\left|\Lambda_{j}\right|\right)
$$

and

$$
\forall \omega \in \Omega_{j, m}: \quad\left\|\frac{f\left(\Lambda_{j}, \omega\right)}{\left|\Lambda_{j}\right|}-f^{*}\right\|_{\infty} \leq 2^{2 d+1}\left(\frac{\left(6 d+3+2 f_{\infty}\right) m^{d}+1}{j-2 m}+\frac{4}{m}\right) .
$$

In particular, almost surely we have $\lim _{n \rightarrow \infty}\left\|\frac{f\left(\Lambda_{n}, \bullet\right)}{\left|\Lambda_{n}\right|}-f^{*}\right\|_{\infty}=0$.

For a precise formulation of the properties of the field $f$, see Section 2. Let us note that in our theorem $f$ takes values in the Banach space $\mathbb{B}$ of right continuous and bounded functions with sup-norm while in $[6,7,11]$ an arbitrary Banach space was allowed. This restriction is due to our use of the Glivenko-Cantelli theory in the proof, and currently we do not know how to extend it to arbitrary Banach spaces.

Naturally, one asks whether the above result and its proof extend to general finitely generated amenable groups. In this case, obviously, the boundary has to be taken with respect to a generating set $S \subseteq G$, and the sequence of squares $\Lambda_{n}$ has to be replaced by a Følner sequence. Indeed, if $G$ satisfies additionally,

$(\boxplus)$ There exists a Følner sequence $\left(\Lambda_{n}\right)_{n \in \mathbb{N}}$ in $G$, and a sequence of symmetric grids $T_{n}=T_{n}^{-1} \subseteq G$ such that $G=\dot{\cup}_{t \in T_{n}} \Lambda_{n} t$ is a disjoint union.

the proofs of [12] apply with technical, but no strategic, modifications, as sketched in Appendix B. 
However, it is not clear in which generality assumption ( $\boxplus$ ) holds. In fact, the existence of tiling Følner sequences (for general amenable groups) has been investigated in several instances. It turned out that there exist useful additional conditions which imply the validity of $(\boxplus)$; cf. [5, 16]. For instance, a group which is residually finite and amenable contains a tiling Følner sequence. Unfortunately, there is a lack of the complete picture: It is still an open question whether there exists a tiling Følner sequence in each amenable group.

Since this question seems hard to answer, Ornstein and Weiss invented in [10] the theory of $\varepsilon$-quasi tilings. The idea is to consider a tiling which is in several senses weaker as the one in $(\boxplus)$. For a given $\varepsilon>0$, one has the following properties:

- the group is not tiled with one element of a Følner sequence, but with finitely many elements of this sequence; the number of these elements depends on $\varepsilon$;

- the tiles are allowed to overlap, but the proportion of the part of any tile which is allowed to intersect other tiles is at most of size $\varepsilon$. This property is called $\varepsilon$-disjointness;

- each element of a Følner sequence with a sufficiently large index is, up to a proportion of size $\varepsilon$ the union of $\varepsilon$-disjoint tiles.

The authors showed that each amenable group can be $\varepsilon$-quasi tiled. In [11], these ideas have been developed further in order to obtain quantitative estimates on the portion which is covered by translates of one specific element of the tiles. The proof of our main result, which we state now in an informal way, is based on these results on quasi tilings.

THEOREM B. Let $\left(\Lambda_{n}\right)$ be a Folner sequence in a finitely generated group $G$. Let $\omega=\left(\omega_{g}\right)_{g \in G} \in \mathbb{R}^{G}$ be an i.i.d. sequence of real random variables. Assume the field

$$
f: \mathcal{P}(G) \times \mathbb{R}^{G} \rightarrow\{D: \mathbb{R} \rightarrow \mathbb{R} \mid D \text { right-continuous and bounded }\},
$$

is $G$-equivariant, monotone in each coordinate $\omega_{g}$, local and almost additive, that is, for disjoint $Q_{1}, \ldots, Q_{n} \subseteq G$ and $Q:=\bigcup_{i=1}^{n} Q_{i}$ we have

$$
\left\|f(Q, \omega)-\sum_{i=1}^{n} f\left(Q_{i}, \omega\right)\right\|_{\infty} \leq \sum_{i=1}^{n}\left|\partial Q_{i}\right|,
$$

where $\partial Q_{i}$ denotes the boundary relative to a set of generators $S \subseteq G$. Assume furthermore, that $f_{\infty}:=\sup _{\omega}\|f(\mathrm{id}, \omega)\|_{\infty}<\infty$.

Then there is a function $f^{*}: \mathbb{R} \rightarrow \mathbb{R}$ such that for each $\delta \in(0,1)$, there exists $a(\delta)>0$, such that for all sufficiently large $j \in \mathbb{N}$, there is an event $\Omega_{j, \delta} \subseteq \mathbb{R}^{G}$, with the properties

$$
\mathbb{P}\left(\Omega_{j, \delta}\right) \geq 1-\exp \left(-a(\delta)\left|\Lambda_{j}\right|\right)
$$


and

$$
\forall \omega \in \Omega_{j, \delta} \quad\left\|\frac{f\left(\Lambda_{j}, \omega\right)}{\left|\Lambda_{j}\right|}-f^{*}\right\|_{\infty} \leq\left(37 f_{\infty}+84|S|+131\right) \delta .
$$

In particular, almost surely we have $\lim _{n \rightarrow \infty}\left\|\frac{f\left(\Lambda_{n}, \bullet\right)}{\left|\Lambda_{n}\right|}-f^{*}\right\|_{\infty}=0$.

For a precise formulation, see Definition 2.2 and Theorem 2.5. To achieve the error bound in the theorem, we work with an $\varepsilon$-quasi tiling with $\varepsilon=\delta^{2}$.

REMARK 1.1. Let us sketch the difference between the proof of Theorem B (see also Theorem 2.5 below) and the Theorem 2.8 of [12] sketched as Theorem A above. There we heavily relied on the fact that $\mathbb{Z}^{d}$ can be tiled exactly with any cube of integer length. Since a general discrete amenable group need not have such a tiling, we have to modify the geometric parts of the proof and use $\varepsilon$-quasi tilings as in $[10,11]$. Since quasi tilings in general overlap, we lose independence of the corresponding random variables. This requires a change in the probabilistic part of the proof and in particular the use of resampling.

The structure of the paper is as follows. In Section 2, we precisely describe the model and our result. In Section 3, we summarize results about $\varepsilon$-quasi tilings, which are fundamental for our proof. The error estimate in the main theorem and the corresponding approximation procedure naturally split in three parts, which are treated consecutively in Sections 4 to 6 . Section 4 is of geometric nature. Section 5 is based on multivariate Glivenko-Cantelli theory. Section 6 is geometric in spirit again. In the Appendix, we prove a resampling lemma and indicate how the proof of [12] could be adapted to cover monotileable amenable groups.

2. Model and main results. We start this section with the introduction of the geometric and probabilistic setting: We recall the notion of a Cayley graph of an amenable group $G$, introduce random colorings of vertices, and define socalled admissible fields, which are random functions mapping finite subsets of $G$ to functions on $\mathbb{R}$ and satisfying a number of natural properties; cf. Definition 2.2. We are then in the position to formulate our main Theorem 2.5.

Let $G$ be a finitely generated group and $S=S^{-1} \subseteq G \backslash\{\mathrm{id}\}$ a finite generating system. Obviously, $G$ is countable. The set of all finite subsets of $G$ is denoted by $\mathcal{F}$ and is countable as well. Throughout this paper, we will assume that $G$ is amenable, that is, there exists a sequence $\left(\Lambda_{n}\right)_{n \in \mathbb{N}}$ of elements in $\mathcal{F}$ such that for each $K \in \mathcal{F}$ one has

$$
\frac{\left|\Lambda_{n} \triangle K \Lambda_{n}\right|}{\left|\Lambda_{n}\right|} \stackrel{n \rightarrow \infty}{\longrightarrow} 0 .
$$

Here, $K \Lambda_{n}:=\left\{k g \mid k \in K, g \in \Lambda_{n}\right\}$ is the pointwise group multiplication of sets, $\Lambda_{n} \triangle K \Lambda_{n}$ denotes the symmetric difference between the sets $\Lambda_{n}$ and $K \Lambda_{n}$ and $|A|$ 
denotes the cardinality of the finite set $A$. A sequence $\left(\Lambda_{n}\right)_{n \in \mathbb{N}}$ satisfying property (2.1) is called Følner sequence.

The pair $(G, S)$ gives rise to an undirected graph $\Gamma(G, S)=(V, E)$ with vertex set $V:=G$ and edge set $E:=\left\{\{x, y\} \mid x y^{-1} \in S\right\}$. The graph $\Gamma(G, S)$ is known as the Cayley graph of $G$ with respect to the generating system $S$. Note that by symmetry of $S$ the edge set $E$ is well defined. Let $d: G \times G \rightarrow \mathbb{N}_{0}$ denote the usual graph metric of $\Gamma(G, S)$. The distance between two nonempty sets $\Lambda_{1}, \Lambda_{2} \subseteq G$ is given by

$$
d\left(\Lambda_{1}, \Lambda_{2}\right):=\min \left\{d(x, y) \mid x \in \Lambda_{1}, y \in \Lambda_{2}\right\}
$$

In the case where $\Lambda_{1}=\{x\}$ consists of only one element, we write $d\left(x, \Lambda_{2}\right)$ for $d\left(\{x\}, \Lambda_{2}\right)$. The diameter of a nonempty set $\Lambda \in \mathcal{F}$ is defined by $\operatorname{diam}(\Lambda):=$ $\max \{d(x, y) \mid x, y \in \Lambda\}$.

Given $r \geq 0$, the $r$-boundary of a set $\Lambda \subseteq G$ is defined by

$$
\partial^{r}(\Lambda):=\{x \in \Lambda \mid d(x, G \backslash \Lambda) \leq r\} \cup\{x \in G \backslash \Lambda \mid d(x, \Lambda) \leq r\}
$$

and besides this we use the notation:

$$
\Lambda^{r}:=\Lambda \backslash \partial^{r}(\Lambda)=\{x \in \Lambda \mid d(x, G \backslash \Lambda)>r\} .
$$

It is easy to verify that for a given Følner sequence $\left(\Lambda_{n}\right)_{n \in \mathbb{N}}$, or $\left(\Lambda_{n}\right)$ for short, and $r \geq 0$ we have

$$
\lim _{n \rightarrow \infty} \frac{\left|\partial^{r}\left(\Lambda_{n}\right)\right|}{\left|\Lambda_{n}\right|}=0 \quad \text { and } \quad \lim _{n \rightarrow \infty} \frac{\left|\Lambda_{n}^{r}\right|}{\left|\Lambda_{n}\right|}=1 .
$$

Moreover, if $\left(\Lambda_{n}\right)$ is a Følner sequence, then for arbitrary $r \geq 0$ the sequence $\left(\Lambda_{n}^{r}\right)$ is a Følner sequence as well. Conversely, in order to show that a given sequence $\left(\Lambda_{n}\right)$ is a Følner sequence, it is sufficient $[1,13]$ to show for $n \rightarrow \infty$ either

$$
\frac{\left|\Lambda_{n} \Delta S \Lambda_{n}\right|}{\left|\Lambda_{n}\right|} \rightarrow 0 \quad \text { or } \quad \frac{\left|\partial^{1}\left(\Lambda_{n}\right)\right|}{\left|\Lambda_{n}\right|} \rightarrow 0 .
$$

Let us introduce colorings of the group $G$ [or equivalently colorings of the vertices of $\Gamma(G, S)]$. We choose a (finite or infinite) set of possible colors $\mathcal{A} \in \mathcal{B}(\mathbb{R})$. The sample set,

$$
\Omega=\mathcal{A}^{G}=\left\{\omega=\left(\omega_{g}\right)_{g \in G} \mid \omega_{j} \in \mathcal{A}\right\},
$$

is the set of all possible colorings of $G$. Note that $G$ acts in a natural way via translations on $\Omega$. To be precise, we define for each $g \in G$

$$
\tau_{g}: \Omega \rightarrow \Omega, \quad\left(\tau_{g} \omega\right)_{x}=\omega_{x g} \quad(x \in G) .
$$

Next, we introduce random colorings. As the $\sigma$-algebra, we choose $\mathcal{B}(\Omega)$, the product $\sigma$-algebra on $\Omega$ generated by cylinder sets. Oftentimes, we are interested 
in (finite) products of $\mathcal{A}$ embedded in the infinite product space $\Omega$. To this end, we set for $\Lambda \subseteq G$

$$
\Omega_{\Lambda}:=\mathcal{A}^{\Lambda}:=\left\{\left(\omega_{g}\right)_{g \in \Lambda} \mid \omega_{g} \in \mathcal{A}\right\}
$$

and define

$$
\Pi_{\Lambda}: \Omega \rightarrow \Omega_{\Lambda} \quad \text { by } \quad\left(\Pi_{\Lambda}(\omega)\right)_{g}:=\omega_{g} \quad \text { for each } g \in \Lambda .
$$

As shorthand notation, we write $\omega_{\Lambda}$ instead of $\Pi_{\Lambda}(\omega)$. Having introduced the measurable space $(\Omega, \mathcal{B}(\Omega))$, we choose a probability measure $\mathbb{P}$ with the following properties:

(M1) equivariance: For each $g \in G$, we have $\mathbb{P} \circ \tau_{g}^{-1}=\mathbb{P}$.

(M2) existence of densities: There is a $\sigma$-finite measure $\mu_{0}$ on $(\mathcal{A}, \mathcal{B}(\mathcal{A}))$, such that for each $\Lambda \in \mathcal{F}$ the measure $\mathbb{P}_{\Lambda}:=\mathbb{P} \circ \Pi_{\Lambda}^{-1}$ is absolutely continuous with respect to $\mu_{\Lambda}:=\bigotimes_{g \in \Lambda} \mu_{0}$ on $\Omega_{\Lambda}$. We denote the corresponding probability density function by $\rho_{\Lambda}$.

(M3) independence condition: There exists $r \geq 0$ such that for all $n \in \mathbb{N}$ and nonempty $\Lambda_{1}, \ldots, \Lambda_{n} \in \mathcal{F}$ with $\min \left\{d\left(\Lambda_{i}, \Lambda_{j}\right) \mid i \neq j\right\}>r$ we have $\rho_{\Lambda}=$ $\prod_{j=1}^{n} \rho_{\Lambda_{j}}$, where $\Lambda=\bigcup_{j=1}^{n} \Lambda_{j}$.

The measure $\mathbb{P}_{\Lambda}$ is called the marginal measure of $\mathbb{P}$. It is defined on $\left(\Omega_{\Lambda}, \mathcal{B}\left(\Omega_{\Lambda}\right)\right)$, where again $\mathcal{B}\left(\Omega_{\Lambda}\right)$ is generated by the corresponding cylinder sets.

REMARK 2.1. (a) The constant $r \geq 0$ in (M3) can be interpreted as the correlation length. In particular, if $r=0$ this property implies that the colors of the vertices are chosen independently.

(b) (M2) is trivially satisfied, if $\mathbb{P}$ is a product measure.

In the following, we consider partial orderings on $\Omega$ and on $\mathbb{R}^{k}$, respectively. Here, we write $\omega \leq \omega^{\prime}$ for $\omega, \omega^{\prime} \in \Omega$, if for all $g \in G$ we have $\omega_{g} \leq \omega_{g}^{\prime}$. The notion $x \leq x^{\prime}$ for $x, x^{\prime} \in \mathbb{R}^{k}$ is defined in the same way. We consider the Banach space

$$
\mathbb{B}:=\{F: \mathbb{R} \rightarrow \mathbb{R} \mid F \text { right-continuous and bounded }\},
$$

which is equipped with supremum norm $\|\cdot\|:=\|\cdot\|_{\infty}$.

Definition 2.2. A field $f: \mathcal{F} \times \Omega \rightarrow \mathbb{B}$ is called admissible if the following conditions are satisfied:

(A1) equivariance: for $\Lambda \in \mathcal{F}, g \in G$ and $\omega \in \Omega$ we have

$$
f(\Lambda g, \omega)=f\left(\Lambda, \tau_{g} \omega\right) .
$$
have

(A2) locality: for all $\Lambda \in \mathcal{F}$ and $\omega, \omega^{\prime} \in \Omega$ satisfying $\Pi_{\Lambda}(\omega)=\Pi_{\Lambda}\left(\omega^{\prime}\right)$ we

$$
f(\Lambda, \omega)=f\left(\Lambda, \omega^{\prime}\right)
$$


(A3) almost additivity: for arbitrary $\omega \in \Omega$, pairwise disjoint $\Lambda_{1}, \ldots, \Lambda_{n} \in \mathcal{F}$ and $\Lambda:=\bigcup_{i=1}^{n} \Lambda_{i}$ we have

$$
\left\|f(\Lambda, \omega)-\sum_{i=1}^{n} f\left(\Lambda_{i}, \omega\right)\right\| \leq \sum_{i=1}^{n} b\left(\Lambda_{i}\right),
$$

where $b: \mathcal{F} \rightarrow[0, \infty)$ satisfies:

- $b(\Lambda)=b(\Lambda g)$ for arbitrary $\Lambda \in \mathcal{F}$ and $g \in G$,

- $\exists D_{f}>0$ with $b(\Lambda) \leq D_{f}|\Lambda|$ for arbitrary $\Lambda \in \mathcal{F}$,

- $\lim _{i \rightarrow \infty} b\left(\Lambda_{i}\right) /\left|\Lambda_{i}\right|=0$, if $\left(\Lambda_{i}\right)_{i \in \mathbb{N}}$ is a Følner sequence.

- for $\Lambda, \Lambda^{\prime} \in \mathcal{F}$, we have $b\left(\Lambda \cup \Lambda^{\prime}\right) \leq b(\Lambda)+b\left(\Lambda^{\prime}\right), b\left(\Lambda \cap \Lambda^{\prime}\right) \leq b(\Lambda)+b\left(\Lambda^{\prime}\right)$, and $b\left(\Lambda \backslash \Lambda^{\prime}\right) \leq b(\Lambda)+b\left(\Lambda^{\prime}\right)$.

(A4) monotonicity: $f$ is antitone with respect to the partial orderings on $\Omega \subseteq$ $\mathbb{R}^{G}$ and $\mathbb{B}$, that is, if $\omega, \omega^{\prime} \in \Omega$ satisfy $\omega \leq \omega^{\prime}$, we have

$$
f(\Lambda, \omega)(x) \geq f\left(\Lambda, \omega^{\prime}\right)(x) \quad \text { for all } x \in \mathbb{R} \text { and } \Lambda \in \mathcal{F} .
$$

(A5) boundedness:

$$
\sup _{\omega \in \Omega}\|f(\{\operatorname{id}\}, \omega)\|<\infty .
$$

REMARK 2.3. - Locality (A2) can be formulated as follows: $f(\Lambda, \cdot)$ is $\sigma\left(\Pi_{\Lambda}\right)$-measurable. This enables us to define $f_{\Lambda}: \Omega_{\Lambda} \rightarrow \mathbb{B}$ by $f_{\Lambda}\left(\omega_{\Lambda}\right):=$ $f(\Lambda, \omega)$ with $\Lambda \in \mathcal{F}$ and $\omega \in \Omega$.

- We call the function $b$ in (A3) boundary term. Note that the fourth assumption on $b$ in (A3) was not made in [12]. Indeed, this inequality is used to separate overlapping tiles and is unnecessary as soon as the group has the tiling property $(\boxplus)$. This fourth point is used only in Lemmas 3.5 and 5.3.

- The antitonicity assumption in (A4) can be weakened. In particular, our proofs apply to fields which are monotone in each coordinate, where the direction of the monotonicity can be different for distinct coordinates. For simplicity reasons and as our main example (see [12]) satisfies (A4), we restrict ourselves to this kind of monotonicity.

- As shown in [12], a combination of (A1), (A3) and (A5) implies that the bound

$$
\begin{aligned}
K_{f} & :=\sup \{\|f(\Lambda, \omega)\| /|\Lambda| \mid \omega \in \Omega, \Lambda \in \mathcal{F}\} \\
& \leq D_{f}+\sup _{\omega \in \Omega}\|f(\{\mathrm{id}\}, \omega)\|<\infty .
\end{aligned}
$$

DEFinition 2.4. A set $\mathcal{U}$ of admissible fields is called admissible set, if their bound is uniform:

$$
K_{\mathcal{U}}:=\sup _{f \in \mathcal{U}} K_{f}<\infty
$$

and each for each $f \in \mathcal{U}$ condition (A3) is satisfied with the same boundary term $b$. In this situation, we denote the constant in (A3) by $D_{\mathcal{U}}$. 
Let us state the main theorem of this paper.

THEOREM 2.5. Let $G$ be a finitely generated amenable group with a Følner sequence $\left(\Lambda_{n}\right)$. Further, let $\mathcal{A} \in \mathcal{B}(\mathbb{R})$ and $\left(\Omega=\mathcal{A}^{G}, \mathcal{B}(\Omega), \mathbb{P}\right)$ a probability space such that $\mathbb{P}$ satisfies (M1) to (M3). Finally, let $\mathcal{U}$ be an admissible set.

(a) Then there exists an event $\tilde{\Omega} \in \mathcal{B}(\Omega)$ such that $\mathbb{P}(\tilde{\Omega})=1$ and for any $f \in \mathcal{U}$ there exists a function $f^{*} \in \mathbb{B}$, which does not depend on the specific Folner seqeunce $\left(\Lambda_{n}\right)$, with

$$
\forall \omega \in \tilde{\Omega}: \quad \lim _{n \rightarrow \infty}\left\|\frac{f\left(\Lambda_{n}, \omega\right)}{\left|\Lambda_{n}\right|}-f^{*}\right\|=0 .
$$

(b) Furthermore, for each $\varepsilon \in(0,1 / 10)$, there exist $j_{0}(\varepsilon) \in \mathbb{N}$, independent of $K_{\mathcal{U}}$, and $a\left(\varepsilon, K_{\mathcal{U}}\right), b\left(\varepsilon, K_{\mathcal{U}}\right)>0$, such that for all $j \in \mathbb{N}, j \geq j_{0}(\varepsilon)$, there is an event $\Omega_{j, \varepsilon, K_{\mathcal{U}}} \in \mathcal{B}(\Omega)$, with the properties

$$
\mathbb{P}\left(\Omega_{j, \varepsilon, K_{\mathcal{U}}}\right) \geq 1-b\left(\varepsilon, K_{\mathcal{U}}\right) \exp \left(-a\left(\varepsilon, K_{\mathcal{U}}\right)\left|\Lambda_{j}\right|\right)
$$

and

$$
\begin{aligned}
& \left\|\frac{f\left(\Lambda_{j}, \omega\right)}{\left|\Lambda_{j}\right|}-f^{*}\right\| \\
& \quad \leq\left(37 K_{\mathcal{U}}+47 D_{\mathcal{U}}+47\right) \sqrt{\varepsilon} \quad \text { for all } \omega \in \Omega_{j, \varepsilon, K_{\mathcal{U}}} \text { and all } f \in \mathcal{U} .
\end{aligned}
$$

For examples of measures $\mathbb{P}$ satisfying (M1) to (M3) and of admissible fields, we refer to [12]. The generalization of the geometry from the lattice $\mathbb{Z}^{d}$ to an amenable group $G$ does not affect the examples. See also $[9,15]$ for a discussion of models giving rise to a discontinuous integrated density of states, which nevertheless can be uniformly approximated by almost additive fields.

3. Outline of $\varepsilon$-quasi tilings. Let us give a brief introduction to the theory of $\varepsilon$-quasi tilings. The main ideas go back to Ornstein and Weiss in [10]. However, the specific results we use here are taken from [11]; see also [13].

Let $\left(Q_{n}\right)$ be a Følner sequence. This sequence is called nested, if for all $n \in \mathbb{N}$ we have $\{\mathrm{id}\} \subseteq Q_{n} \subseteq Q_{n+1}$. Using translations and subsequences, it is easy to show that every amenable group contains a nested Følner sequence; cf. [11], Lemma 2.6.

We will use the elements of the nested Følner sequence $\left(Q_{n}\right)$ to $\varepsilon$-quasi tile elements of a given Følner sequence $\left(\Lambda_{j}\right)$ for (very) large index $j$. The next definition provides the notion of an $\alpha$-covering, $\varepsilon$-disjointness and $\varepsilon$-quasi tiling.

DEFINITION 3.1. Let $G$ be a finitely generated group, $\alpha, \varepsilon \in(0,1)$ and $I$ some index set. 
- The sets $Q_{i} \in \mathcal{F}, i \in I$, are said to $\alpha$-cover the set $\Lambda \in \mathcal{F}$, if:

(i) $\bigcup_{i \in I} Q_{i} \subseteq \Lambda$, and

(ii) $\left|\Lambda \cap \bigcup_{i \in I} Q_{i}\right| \geq \alpha|\Lambda|$.

- The sets $Q_{i} \in \mathcal{F}, i \in I$ are called $\varepsilon$-disjoint, if there are subsets $\stackrel{\circ}{Q}_{i} \subseteq Q_{i}, i \in I$, such that for all $i \in I$ we have:

(i) $\left|Q_{i} \backslash \stackrel{Q}{Q}_{i}\right| \leq \varepsilon\left|Q_{i}\right|$, and

(ii) $\mathscr{Q}_{i}$ and $\bigcup_{j \in I \backslash\{i\}} \stackrel{\circ}{Q}_{j}$ are disjoint.

- The $K_{i} \in \mathcal{F}, i \in I$, are said to $\varepsilon$-quasi tile $\Lambda \in \mathcal{F}$, if there exist $T_{i} \in \mathcal{F}, i \in I$, such that:

(i) the elements of $\left\{K_{i} T_{i} \mid i \in I\right\}$ are pairwise disjoint,

(ii) for each $i \in I$, the elements of $\left\{K_{i} t \mid t \in T_{i}\right\}$ are $\varepsilon$-disjoint, and

(iii) the family $\left\{K_{i} T_{i} \mid i \in I\right\}(1-2 \varepsilon)$-covers $\Lambda$.

The set $T_{i}$ is called center set for the tile $K_{i}, i \in I$.

Actually, the details in this definition are adapted to our needs in this paper, as is the following theorem. The general and more technical versions as well as the proof of can be found [11]. See also [10] for earlier results.

Roughly speaking, the following theorem provides, in the setting of finitely generated amenable groups, $\varepsilon$-quasi covers for every set with small enough boundary compared to its volume. Additionally, the theorem also provides control over the fraction covered by different tiles with uniform almost densities. To quantify these densities, we use the standard notation $\lceil b\rceil:=\inf \{z \in \mathbb{Z} \mid z \geq b\}=\inf \mathbb{Z} \cap[b, \infty)$ for the smallest integer above $b \in \mathbb{R}$ and define, for all $\varepsilon>0$ and $i \in \mathbb{N}$,

$$
N(\varepsilon):=\left\lceil\frac{\ln (\varepsilon)}{\ln (1-\varepsilon)}\right\rceil \text { and } \quad \eta_{i}(\varepsilon):=\varepsilon(1-\varepsilon)^{N(\varepsilon)-i} .
$$

THEOREM 3.2. Let $G$ be a finitely generated amenable group, $\left(Q_{n}\right)$ a nested Folner sequence and $\varepsilon \in(0,1 / 10)$. Then there is a finite and strictly increasing selection of sets $K_{i} \in\left\{Q_{n} \mid n \in \mathbb{N}\right\}, i \in\{1, \ldots, N(\varepsilon)\}$, with the following quasi tiling property. For each Folner sequence $\left(\Lambda_{j}\right)$, there exists $j_{0}(\varepsilon) \in \mathbb{N}$ such that for all $j \geq j_{0}(\varepsilon)$, the sets $K_{i}, i \in\{1, \ldots, N(\varepsilon)\}$, are an $\varepsilon$-quasi tiling of $\Lambda_{j}$. Moreover, for all $j \geq j_{0}(\varepsilon)$ and all $i \in\{1, \ldots, N(\varepsilon)\}$, the proportion of $\Lambda_{j}$ covered by the tile $K_{i}$ satisfies

$$
\left|\frac{\left|K_{i} T_{i}^{j}\right|}{\left|\Lambda_{j}\right|}-\eta_{i}(\varepsilon)\right| \leq \frac{\varepsilon^{2}}{N(\varepsilon)},
$$

where $T_{i}^{j}$ denotes the center set of the tile $K_{i}$ for the $\varepsilon$-quasi cover of $\Lambda_{j}$.

To make full use of Theorem 3.2, we need some properties of the densities $\eta_{i}(\varepsilon)$. 
LEMMA 3.3. For $N(\varepsilon)$ and $\eta_{i}(\varepsilon)$ as in (3.1), the following hold true:

(a) For each $\varepsilon \in(0,1)$, we have

$$
1-\varepsilon \leq \sum_{i=1}^{N(\varepsilon)} \eta_{i}(\varepsilon)=1-(1-\varepsilon)^{N(\varepsilon)} \leq 1 .
$$

(b) For each $\varepsilon \in(0,1 / 10)$ and $i \in\{1, \ldots, N(\varepsilon)\}$, we have

$$
\frac{\varepsilon}{N(\varepsilon)} \leq \eta_{i}(\varepsilon) \leq \varepsilon
$$

(c) For a bounded sequence $\left(\alpha_{i}\right)_{i \in \mathbb{N}}$ and $\varepsilon \in(0,1 / 10)$, we have the inequality

$$
\left|\sum_{i=1}^{N(\varepsilon)} \alpha_{i} \eta_{i}(\varepsilon)\right| \leq A \sqrt{\varepsilon}+A_{\varepsilon}
$$

where $A:=\sup \left\{\left|\alpha_{i}\right| \mid i \in \mathbb{N}\right\}$ and $A_{\varepsilon}:=\sup \left\{\left|\alpha_{i}\right| \mid i \in \mathbb{N}, i \geq \varepsilon^{-1 / 2}\right\}$. In particular,

$$
\lim _{\varepsilon \searrow 0} \sum_{i=1}^{N(\varepsilon)} \alpha_{i} \eta_{i}(\varepsilon) \leq \liminf _{i \rightarrow \infty}\left|\alpha_{i}\right|
$$

PROOF. Part (a) is an easy implication of the sum formula for the geometric series. We refer to [11], Remark 4.3, for the details.

Let us prove (b). By definition of $\eta_{i}(\varepsilon)$, we have $\eta_{i}(\varepsilon) \leq \varepsilon$. In order to see the other inequality, we note that

$$
\eta_{i}(\varepsilon) \geq \varepsilon(1-\varepsilon)^{N(\varepsilon)-1} \geq \varepsilon(1-\varepsilon)^{\frac{\ln (\varepsilon)}{\ln (1-\varepsilon)}}=\varepsilon^{2} .
$$

Thus, it is sufficient to show that $\varepsilon \geq 1 / N(\varepsilon)$. To this end, note that by definition of $N(\varepsilon)$ the following holds true:

$$
\varepsilon N(\varepsilon) \geq \frac{\varepsilon \ln (\varepsilon)}{\ln (1-\varepsilon)} .
$$

Using the assumption $\varepsilon \in(0,1 / 10)$, a short and elementary calculation shows that the last expression is bounded from below by 1 .

To verify part (c), set $N_{\varepsilon}^{*}:=\left\lfloor\varepsilon^{-1 / 2}\right\rfloor:=\sup \mathbb{Z} \cap\left(-\infty, \varepsilon^{-1 / 2}\right]$, and calculate as follows:

$$
\left|\sum_{i=1}^{N(\varepsilon)} \alpha_{i} \eta_{i}(\varepsilon)\right| \leq\left|\sum_{i=1}^{N_{\varepsilon}^{*}} \alpha_{i} \eta_{i}(\varepsilon)\right|+\left|\sum_{i=N_{\varepsilon}^{*}+1}^{N(\varepsilon)} \alpha_{i} \eta_{i}(\varepsilon)\right| \leq A N_{\varepsilon}^{*} \varepsilon+A_{\varepsilon} \leq A \sqrt{\varepsilon}+A_{\varepsilon} .
$$

Note that it is easy to show that for $0<\varepsilon<1 / 10$ we have $N(\varepsilon)>N_{\varepsilon}^{*}>0$, such that both sums are nonempty.

Next, we derive a useful corollary of Theorem 3.2. 
COROLLARY 3.4. Let a finitely generated group $G$, a subset $\Lambda \in \mathcal{F}$ and $\varepsilon \in$ $(0,1 / 2)$ be given. Assume furthermore that the sets $K_{i} \in \mathcal{F}, i \in\{1, \ldots, N(\varepsilon)\}$ are an $\varepsilon$-quasi tiling of $\Lambda$ with almost densities $\eta_{i}(\varepsilon)$ and center sets $T_{i} \in \mathcal{F}$, $i \in\{1, \ldots, N(\varepsilon)\}$, satisfying (3.2). Then we have for each $i \in\{1, \ldots, N(\varepsilon)\}$, the inequality estimating the "density" of the tile $K_{i}$ :

$$
\left|\frac{\left|T_{i}\right|}{|\Lambda|}-\frac{\eta_{i}(\varepsilon)}{\left|K_{i}\right|}\right| \leq 4 \varepsilon \frac{\eta_{i}(\varepsilon)}{\left|K_{i}\right|}
$$

Proof. We fix $i \in\{1, \ldots, N(\varepsilon)\}$, employ $\varepsilon$-disjointness and the density estimate (3.2) and deduce

$$
(1-\varepsilon) \frac{\left|K_{i}\right|\left|T_{i}\right|}{|\Lambda|} \leq \frac{\left|K_{i} T_{i}\right|}{|\Lambda|} \leq \eta_{i}(\varepsilon)+\frac{\varepsilon^{2}}{N(\varepsilon)} .
$$

Therefore, with part (b) of Lemma 3.3, we get

$$
\begin{aligned}
\frac{\left|T_{i}\right|}{|\Lambda|}-\frac{\eta_{i}(\varepsilon)}{\left|K_{i}\right|} & \leq \frac{\eta_{i}(\varepsilon)+\frac{\varepsilon^{2}}{N(\varepsilon)}}{(1-\varepsilon)\left|K_{i}\right|}-\frac{\eta_{i}(\varepsilon)}{\left|K_{i}\right|} \\
& =\frac{\varepsilon \eta_{i}(\varepsilon)+\frac{\varepsilon^{2}}{N(\varepsilon)}}{(1-\varepsilon)\left|K_{i}\right|} \\
& \leq \frac{2 \varepsilon \eta_{i}(\varepsilon)}{(1-\varepsilon)\left|K_{i}\right|} \leq \frac{4 \varepsilon \eta_{i}(\varepsilon)}{\left|K_{i}\right|} .
\end{aligned}
$$

Equation (3.2) gives also a bound for the other direction. To be precise, we use

$$
\eta_{i}(\varepsilon)-\frac{\varepsilon^{2}}{N(\varepsilon)} \leq \frac{\left|K_{i} T_{i}\right|}{|\Lambda|} \leq \frac{\left|K_{i}\right|\left|T_{i}\right|}{|\Lambda|}
$$

and again part (b) of Lemma 3.3 to obtain

$$
\frac{\left|T_{i}\right|}{|\Lambda|}-\frac{\eta_{i}(\varepsilon)}{\left|K_{i}\right|} \geq \frac{\eta_{i}(\varepsilon)-\frac{\varepsilon^{2}}{N(\varepsilon)}}{\left|K_{i}\right|}-\frac{\eta_{i}(\varepsilon)}{\left|K_{i}\right|}=-\frac{\varepsilon^{2}}{N(\varepsilon)\left|K_{i}\right|} \geq-\frac{\varepsilon \eta_{i}(\varepsilon)}{\left|K_{i}\right|}
$$

This implies the claimed bound.

Finally, we provide a generalization of almost additivity for sets which are not disjoint, but only $\varepsilon$-disjoint. The proof can be found in [13], Lemma 5.23.

LEMMA 3.5. Let $G$ be a finitely generated group, $f$ an admissible field with boundary term $b$ and $\varepsilon \in(0,1 / 2)$. Then for any $\varepsilon$-disjoint sets $Q_{i}, i \in\{1, \ldots, k\}$, we have for each $\omega \in \Omega$

$$
\left\|f(Q, \omega)-\sum_{i=1}^{k} f\left(Q_{i}, \omega\right)\right\| \leq \varepsilon\left(3 K_{f}+9 D_{f}\right)|Q|+3 \sum_{i=1}^{k} b\left(Q_{i}\right),
$$

where $Q:=\bigcup_{i=1}^{k} Q_{i}$ and $D_{f}$ is the constant from (A3) of Definition 2.2. 
4. Approximation via the empirical measure. Given some Følner sequence $\left(\Lambda_{j}\right)$ and an admissible field $f$, the aim of this section is the approximation of the expression $\frac{f\left(\Lambda_{j}, \omega\right)}{\left|\Lambda_{j}\right|}$ using elements of a second Følner sequence $\left(Q_{n}\right)$ and associated empirical measures; cf. Lemma 4.3. This second sequence needs to satisfy certain additional assumptions, namely we need that $\left(Q_{n}\right)$ is nested and satisfies for the correlation length $r \in \mathbb{N}_{0}$ from (M3) that the sequences

$$
\begin{array}{ll}
\left(\frac{b\left(Q_{n}\right)}{\left|Q_{n}\right|}\right), & \left(\frac{b\left(Q_{n}^{r}\right)}{\left|Q_{n}\right|}\right) \text { and } \\
\left(\frac{\left|\partial^{r}\left(Q_{n}\right)\right|}{\left|Q_{n}\right|}\right) & \text { converge monotonically to } 0 .
\end{array}
$$

That these sequences converge to zero is clear by the fact that $\left(Q_{n}\right)$ is a Følner sequence and $b$ a boundary term in the sense of Definition 2.2. In order to obtain the monotonicity in (4.1), we choose a subsequence of $\left(Q_{n}\right)$. These considerations show that each amenable group admits a nested Følner sequence $\left(Q_{n}\right)$ which satisfies (4.1). These terms will be used in the error estimates in the approximations throughout this text. To abbreviate the notation, we define

$$
\beta_{n}^{\prime}:=\max \left\{\frac{b\left(Q_{n}\right)}{\left|Q_{n}\right|}, \frac{b\left(Q_{n}^{r}\right)}{\left|Q_{n}\right|}, \frac{\left|\partial^{r}\left(Q_{n}\right)\right|}{\left|Q_{n}\right|}\right\} \quad \text { and } \quad \beta(\varepsilon):=\beta_{1}^{\prime} \sqrt{\varepsilon}+\beta_{\lceil 1 / \sqrt{\varepsilon}\rceil}^{\prime}
$$

for $n \in \mathbb{N}$ and $\varepsilon \in(0,1 / 10)$. Note that $\left(\beta_{n}^{\prime}\right)_{n}$ is a monotone sequence and converges to 0 , and that by Lemma 3.3(c)

$$
\sum_{i=1}^{N(\varepsilon)} \beta_{i}^{\prime} \eta_{i}(\varepsilon) \leq \beta(\varepsilon) \stackrel{\varepsilon \searrow 0}{\longrightarrow} 0 .
$$

REMARK 4.1. For the proof of Theorem 2.5, we additionally have to ensure $\beta_{n}^{\prime} \leq(2 n)^{-1}$ for all $n \in \mathbb{N}$ while taking the subsequences above. We will track the boundary terms throughout the paper and use $\beta(\varepsilon)$ until the very end, where we simplify the result by applying

$$
\beta(\varepsilon)=\beta_{1}^{\prime} \sqrt{\varepsilon}+\beta_{\lceil 1 / \sqrt{\varepsilon}\rceil}^{\prime} \leq \frac{1}{2} \sqrt{\varepsilon}+\frac{1}{2\lceil 1 / \sqrt{\varepsilon}\rceil} \leq \sqrt{\varepsilon} .
$$

The cost of this additional condition on the boundary terms is that, via Theorem 3.2, $j_{0}(\varepsilon)$ in Theorem 2.5 will potentially increase. But up to here, we deal only with the geometry of $G$ and still have that $j_{0}(\varepsilon)$ depends only on $\varepsilon$.

Moreover, let us emphasize that when considering an admissible set $\mathcal{U}$ the value $\sqrt{\varepsilon}$ gives a uniform bound on $\beta(\varepsilon)$ for all $f \in \mathcal{U}$, since in this situation all $f \in \mathcal{U}$ are almost additive with the same boundary term $b$.

Define for an admissible field $f$ and $\Lambda \in \mathcal{F}$ the function

$$
f_{\Lambda}: \Omega_{\Lambda} \rightarrow \mathbb{B}, f_{\Lambda}(\omega):=f\left(\Lambda, \omega^{\prime}\right) \quad \text { where } \omega^{\prime} \in \Pi_{\Lambda}^{-1}(\{\omega\}) .
$$


Note that by (A2) of Definition 2.2 we see that $f_{\Lambda}$ is well defined (and measurable). In the situation where we insert elements of the Følner sequence $\left(\Lambda_{n}\right)$ or $\left(\Lambda_{n}^{r}\right)$, for some $r \in \mathbb{N}_{0}$, we write

$$
f_{n}:=f_{\Lambda_{n}} \quad \text { or } \quad f_{n}^{r}:=f_{\Lambda_{n}^{r}} .
$$

For given $K, T \in \mathcal{F}$ and $\omega \in \Omega$, we define the empirical measure by

$$
L^{\omega}(K, T): \mathcal{B}\left(\Omega_{K T}\right) \rightarrow[0,1], \quad L^{\omega}(K, T)=\frac{1}{|T|} \sum_{t \in T} \delta_{\left(\tau_{t} \omega\right)_{K}} .
$$

Given $\varepsilon \in(0,1 / 10)$ and sequences $\left(\Lambda_{j}\right)$ and $\left(Q_{j}\right)$ as above, we obtain by Theorem 3.2 finite sets $K_{i}(\varepsilon), i=1, \ldots, N(\varepsilon)$ and (for $j$ large enough) center sets $T_{i}^{j}(\varepsilon)$ which form an $\varepsilon$-quasi tiling of $\Lambda_{j}$. In this setting, we use for given $\omega \in \Omega$, $\varepsilon \in(0,1 / 10), r>0, i \in\{1, \ldots, N(\varepsilon)\}$ and $j \in \mathbb{N}$ large enough the notation

$$
L_{i, j}^{\omega}(\varepsilon):=L^{\omega}\left(K_{i}(\varepsilon), T_{i}^{j}(\varepsilon)\right) \text { and } f_{i}(\varepsilon):=f_{K_{i}(\varepsilon)}
$$

as well as

$$
L_{i, j}^{r, \omega}(\varepsilon):=L^{\omega}\left(K_{i}^{r}(\varepsilon), T_{i}^{j}(\varepsilon)\right) \quad \text { and } \quad f_{i}^{r}(\varepsilon):=f_{K_{i}^{r}(\varepsilon)} .
$$

Here, the reader may recall that $K_{i}^{r}(\varepsilon)=K_{i}(\varepsilon) \backslash \partial^{r}\left(K_{i}(\varepsilon)\right)$.

Moreover, we use for $\Lambda \in \mathcal{F}$, a measurable $f: \Omega_{\Lambda} \rightarrow \mathbb{B}$ and a measure $v$ on $\left(\Omega_{\Lambda}, \mathcal{B}\left(\Omega_{\Lambda}\right)\right)$ the notation

$$
\langle f, v\rangle:=\int_{\Omega_{\Lambda}} f(\omega) \mathrm{d} v(\omega)
$$

LEMMA 4.2. Let $f$ be an admissible field and let $K, T \in \mathcal{F}$ and $\omega \in \Omega$. Then

$$
\left\langle f_{K}, L^{\omega}(K, T)\right\rangle=\frac{1}{|T|} \sum_{t \in T} f(K t, \omega) .
$$

PROOF. We calculate using linearity and (A1) of Definition 2.2:

$$
\begin{aligned}
\left\langle f_{K}, L^{\omega}(K, T)\right\rangle & =\int_{\Omega_{K}} f_{K}\left(\omega^{\prime}\right) \mathrm{d} L^{\omega}(K, T)\left(\omega^{\prime}\right) \\
& =\frac{1}{|T|} \sum_{t \in T} \int_{\Omega_{K}} f_{K}\left(\omega^{\prime}\right) \mathrm{d} \delta_{\left(\tau_{t} \omega\right)_{K}}\left(\omega^{\prime}\right) \\
& =\frac{1}{|T|} \sum_{t \in T} f_{K}\left(\left(\tau_{t} \omega\right)_{K}\right) \\
& =\frac{1}{|T|} \sum_{t \in T} f(K t, \omega) .
\end{aligned}
$$

We proceed with the first approximation lemma. 
LEMMA 4.3. Let $G$ be a finitely generated amenable group, let $f$ be an admissible field and let $\left(\Lambda_{n}\right)$ and $\left(Q_{n}\right)$ be Folner sequences, where $\left(Q_{n}\right)$ is additionally nested and satisfies (4.1). Then we have for all $\omega \in \Omega$ that

$$
\lim _{\varepsilon \searrow 0} \lim _{j \rightarrow \infty}\left\|\frac{f\left(\Lambda_{j}, \omega\right)}{\left|\Lambda_{j}\right|}-\sum_{i=1}^{N(\varepsilon)} \eta_{i}(\varepsilon) \frac{\left\langle f_{i}^{r}(\varepsilon), L_{i, j}^{r, \omega}(\varepsilon)\right\rangle}{\left|K_{i}(\varepsilon)\right|}\right\|=0,
$$

where $K_{i}(\varepsilon), i \in\{1, \ldots, N(\varepsilon)\}$ are given by Theorem 3.2. Moreover, we have for arbitrary $\varepsilon \in(0,1 / 10)$ and $j \geq j_{0}(\varepsilon)$, with $j_{0}(\varepsilon)$ from Theorem 3.2, the inequality

$$
\begin{aligned}
& \left\|\frac{f\left(\Lambda_{j}, \omega\right)}{\left|\Lambda_{j}\right|}-\sum_{i=1}^{N(\varepsilon)} \eta_{i}(\varepsilon) \frac{\left\langle f_{i}^{r}(\varepsilon), L_{i, j}^{r, \omega}(\varepsilon)\right\rangle}{\left|K_{i}(\varepsilon)\right|}\right\| \\
& \quad \leq\left(9 K_{f}+15 D_{f}\right) \varepsilon+12\left(2+K_{f}+D_{f}\right) \beta(\varepsilon) .
\end{aligned}
$$

ProOF. Let $\varepsilon \in(0,1 / 10)$ and $j \geq j_{0}(\varepsilon) \in \mathbb{N}$ be given, where $j_{0}(\varepsilon)$ is the constant given by Theorem 3.2. We estimate using the triangle inequality

$$
\begin{gathered}
\left\|\frac{f\left(\Lambda_{j}, \omega\right)}{\left|\Lambda_{j}\right|}-\sum_{i=1}^{N(\varepsilon)} \eta_{i}(\varepsilon) \frac{\left\langle f_{i}^{r}(\varepsilon), L_{i, j}^{r, \omega}(\varepsilon)\right\rangle}{\left|K_{i}(\varepsilon)\right|}\right\| \\
\leq a(\varepsilon, j)+\sum_{i=1}^{N(\varepsilon)} b_{i}(\varepsilon, j)+\sum_{i=1}^{N(\varepsilon)} c_{i}(\varepsilon, j),
\end{gathered}
$$

where

$$
\begin{aligned}
& a(\varepsilon, j):=\frac{1}{\left|\Lambda_{j}\right|}\left\|f\left(\Lambda_{j}, \omega\right)-\sum_{i=1}^{N(\varepsilon)} \sum_{t \in T_{i}^{j}(\varepsilon)} f\left(K_{i}(\varepsilon) t, \omega\right)\right\|, \\
& b_{i}(\varepsilon, j):=\left\|\sum_{t \in T_{i}^{j}(\varepsilon)} \frac{f\left(K_{i}(\varepsilon) t, \omega\right)}{\left|\Lambda_{j}\right|}-\eta_{i}(\varepsilon) \frac{\left\langle f_{i}(\varepsilon), L_{i, j}^{\omega}(\varepsilon)\right\rangle}{\left|K_{i}(\varepsilon)\right|}\right\| \quad \text { and } \\
& c_{i}(\varepsilon, j):=\frac{\eta_{i}(\varepsilon)}{\left|K_{i}(\varepsilon)\right|}\left\|\left\langle f_{i}(\varepsilon), L_{i, j}^{\omega}(\varepsilon)\right\rangle-\left\langle f_{i}^{r}(\varepsilon), L_{i, j}^{r, \omega}(\varepsilon)\right\rangle\right\| .
\end{aligned}
$$

Here, the expressions $L_{i, j}^{\omega}(\varepsilon)$ and $f_{i}(\varepsilon)$ are given by (4.7). Let us estimate the term $a(\varepsilon, j)$. To this end, denote the part which is covered by translates of $K_{i}(\varepsilon)$, $i \in\{1, \ldots, N(\varepsilon)\}$ by

$$
R_{i}^{j}(\varepsilon):=\bigcup_{i=1}^{N(\varepsilon)} K_{i}(\varepsilon) T_{i}^{j}(\varepsilon) \subseteq \Lambda_{j}
$$

Then we have, using the properties of the $\varepsilon$-quasi tiling and part (a) of Lemma 3.3,

$$
\left|R_{i}^{j}(\varepsilon)\right|=\sum_{i=1}^{N(\varepsilon)}\left|K_{i}(\varepsilon) T_{i}^{j}(\varepsilon)\right| \geq\left|\Lambda_{j}\right| \sum_{i=1}^{N(\varepsilon)}\left(\eta_{i}(\varepsilon)-\frac{\varepsilon^{2}}{N(\varepsilon)}\right) \geq(1-2 \varepsilon)\left|\Lambda_{j}\right|,
$$


which in turn gives $\left|\Lambda_{j} \backslash R_{i}^{j}(\varepsilon)\right| \leq 2 \varepsilon\left|\Lambda_{j}\right|$. We use this and Lemma 3.5 to calculate

$$
\begin{aligned}
\left|\Lambda_{j}\right| a(\varepsilon, j) \leq & \left(3 K_{f}+9 D_{f}\right) \varepsilon\left|\Lambda_{j}\right|+3 b\left(\Lambda_{j} \backslash R_{i}^{j}(\varepsilon)\right) \\
& +\left\|f\left(\Lambda_{j} \backslash R_{i}^{j}(\varepsilon)\right)\right\|+3 \sum_{i=1}^{N(\varepsilon)} \sum_{t \in T_{i}^{j}(\varepsilon)} b\left(K_{i}(\varepsilon) t\right) \\
\leq & \left(3 K_{f}+9 D_{f}\right) \varepsilon\left|\Lambda_{j}\right|+\left(K_{f}+3 D_{f}\right)\left|\Lambda_{j} \backslash R_{i}^{j}(\varepsilon)\right| \\
& +3 \sum_{i=1}^{N(\varepsilon)}\left|T_{i}^{j}(\varepsilon)\right| b\left(K_{i}(\varepsilon)\right) \\
\leq & \left(5 K_{f}+15 D_{f}\right) \varepsilon\left|\Lambda_{j}\right|+3 \sum_{i=1}^{N(\varepsilon)}\left|T_{i}^{j}(\varepsilon)\right| b\left(K_{i}(\varepsilon)\right) .
\end{aligned}
$$

By $\varepsilon$-disjointness and (3.2), we obtain

$$
\begin{aligned}
\frac{1}{2}\left|K_{i}(\varepsilon)\right|\left|T_{i}^{j}(\varepsilon)\right| & \leq(1-\varepsilon)\left|K_{i}(\varepsilon)\right|\left|T_{i}^{j}(\varepsilon)\right| \\
& \leq\left|K_{i}(\varepsilon) T_{i}^{j}(\varepsilon)\right| \\
& \leq\left(\eta_{i}(\varepsilon)+\frac{\varepsilon^{2}}{N(\varepsilon)}\right)\left|\Lambda_{j}\right|,
\end{aligned}
$$

which together with (b) of Lemma 3.3 gives

$$
\begin{aligned}
& \sum_{i=1}^{N(\varepsilon)}\left|T_{i}^{j}(\varepsilon)\right| b\left(K_{i}(\varepsilon)\right) \\
& \quad \leq 2\left|\Lambda_{j}\right| \sum_{i=1}^{N(\varepsilon)}\left(\eta_{i}(\varepsilon)+\frac{\varepsilon^{2}}{N(\varepsilon)}\right) \frac{b\left(K_{i}(\varepsilon)\right)}{\left|K_{i}(\varepsilon)\right|} \\
& \quad \leq 4\left|\Lambda_{j}\right| \sum_{i=1}^{N(\varepsilon)} \eta_{i}(\varepsilon) \frac{b\left(K_{i}(\varepsilon)\right)}{\left|K_{i}(\varepsilon)\right|} .
\end{aligned}
$$

This implies the following bound:

$$
a(\varepsilon, j) \leq\left(5 K_{f}+15 D_{f}\right) \varepsilon+12 \sum_{i=1}^{N(\varepsilon)} \eta_{i}(\varepsilon) \frac{b\left(K_{i}(\varepsilon)\right)}{\left|K_{i}(\varepsilon)\right|} .
$$

To estimate the second term in (4.10), we apply Lemma 4.2 to obtain

$$
\sum_{t \in T_{i}^{j}(\varepsilon)} f\left(K_{i}(\varepsilon) t, \omega\right)=\left|T_{i}^{j}(\varepsilon)\right| \cdot\left\langle f_{i}(\varepsilon), L_{i, j}^{\omega}(\varepsilon)\right\rangle .
$$


Thus, by Corollary 3.4 and the fact $\left\|\left\langle f_{i}(\varepsilon), L_{i, j}^{\omega}(\varepsilon)\right\rangle\right\| \leq K_{f}\left|K_{i}(\varepsilon)\right|$, we have for each $i \in\{1, \ldots, N(\varepsilon)\}$

$$
\begin{aligned}
b_{i}(\varepsilon, j) & =\left\|\frac{\left|T_{i}^{j}(\varepsilon)\right|\left\langle f_{i}(\varepsilon), L_{i, j}^{\omega}(\varepsilon)\right\rangle}{\left|\Lambda_{j}\right|}-\eta_{i}(\varepsilon) \frac{\left\langle f_{i}(\varepsilon), L_{i, j}^{\omega}(\varepsilon)\right\rangle}{\left|K_{i}(\varepsilon)\right|}\right\| \\
& =\left|\frac{\left|T_{i}^{j}(\varepsilon)\right|}{\left|\Lambda_{j}\right|}-\frac{\eta_{i}(\varepsilon)}{\left|K_{i}(\varepsilon)\right|}\right|\left\|\left\langle f_{i}(\varepsilon), L_{i, j}^{\omega}(\varepsilon)\right\rangle\right\| \\
& \leq 4 \frac{\varepsilon \eta_{i}(\varepsilon)}{\left|K_{i}(\varepsilon)\right|} K_{f}\left|K_{i}(\varepsilon)\right|=4 K_{f} \varepsilon \eta_{i}(\varepsilon) .
\end{aligned}
$$

Let us finally estimate the term $c_{i}(\varepsilon, j)$. By Lemma 4.2, we have for each $i \in$ $\{1, \ldots, N(\varepsilon)\}$ :

$$
\begin{aligned}
\left\|\left\langle f_{i}(\varepsilon), L_{i, j}^{\omega}(\varepsilon)\right\rangle-\left\langle f_{i}^{r}(\varepsilon), L_{i, j}^{r, \omega}(\varepsilon)\right\rangle\right\| \\
\leq \frac{1}{\left|T_{i}^{j}(\varepsilon)\right|} \sum_{t \in T_{i}^{j}(\varepsilon)}\left\|f\left(K_{i}(\varepsilon) t, \omega\right)-f\left(K_{i}^{r}(\varepsilon) t, \omega\right)\right\| \\
\leq \frac{1}{\left|T_{i}^{j}(\varepsilon)\right|} \sum_{t \in T_{i}^{j}(\varepsilon)} b\left(K_{i}^{r}(\varepsilon)\right)+b\left(\partial^{r}\left(K_{i}(\varepsilon)\right) \cap K_{i}(\varepsilon)\right) \\
\quad+\left\|f\left(\partial^{r}\left(K_{i}(\varepsilon)\right) t \cap K_{i}(\varepsilon) t, \omega\right)\right\| \\
\leq b\left(K_{i}^{r}(\varepsilon)\right)+\left(K_{f}+D_{f}\right)\left|\partial^{r}\left(K_{i}(\varepsilon)\right)\right| .
\end{aligned}
$$

Together with (4.10), the estimates for $a(\varepsilon, j)$ in (4.12), for $b_{i}(\varepsilon, j)$ in (4.13) and for $c_{i}(\varepsilon, j)$ in (4.14) yield

$$
\begin{aligned}
\left\|\frac{f\left(\Lambda_{j}, \omega\right)}{\left|\Lambda_{j}\right|}-\sum_{i=1}^{N(\varepsilon)} \eta_{i}(\varepsilon) \frac{\left\langle f_{i}^{r}(\varepsilon), L_{i, j}^{r, \omega}(\varepsilon)\right\rangle}{\left|K_{i}(\varepsilon)\right|}\right\| \\
\leq \quad\left(5 K_{f}+15 D_{f}\right) \varepsilon+12 \sum_{i=1}^{N(\varepsilon)} \eta_{i}(\varepsilon) \frac{b\left(K_{i}(\varepsilon)\right)}{\left|K_{i}(\varepsilon)\right|} \\
\quad+\sum_{i=1}^{N(\varepsilon)} \eta_{i}(\varepsilon)\left(4 K_{f} \varepsilon+\frac{b\left(K_{i}^{r}(\varepsilon)\right)+\left(K_{f}+D_{f}\right)\left|\partial^{r}\left(K_{i}(\varepsilon)\right)\right|}{\left|K_{i}(\varepsilon)\right|}\right) \\
\leq \quad\left(9 K_{f}+15 D_{f}\right) \varepsilon \\
\quad+12 \sum_{i=1}^{N(\varepsilon)} \eta_{i}(\varepsilon) \frac{b\left(K_{i}(\varepsilon)\right)+b\left(K_{i}^{r}(\varepsilon)\right)+\left(K_{f}+D_{f}\right)\left|\partial^{r}\left(K_{i}(\varepsilon)\right)\right|}{\left|K_{i}(\varepsilon)\right|} .
\end{aligned}
$$


To verify (4.9), recall that we assumed that $\left(Q_{n}\right)$ satisfies (4.1). By the choice of $K_{i}(\varepsilon)$ in Theorem 3.2, this gives

$$
\begin{aligned}
& \left\|\frac{f\left(\Lambda_{j}, \omega\right)}{\left|\Lambda_{j}\right|}-\sum_{i=1}^{N(\varepsilon)} \eta_{i}(\varepsilon) \frac{\left\langle f_{i}^{r}(\varepsilon), L_{i, j}^{r, \omega}(\varepsilon)\right\rangle}{\left|K_{i}(\varepsilon)\right|}\right\| \\
& \leq\left(9 K_{f}+15 D_{f}\right) \varepsilon \\
& \quad+12 \sum_{i=1}^{N(\varepsilon)} \eta_{i}(\varepsilon) \underbrace{\frac{b\left(Q_{i}\right)+b\left(Q_{i}^{r}\right)+\left(K_{f}+D_{f}\right)\left|\partial^{r}\left(Q_{i}\right)\right|}{\left|Q_{i}\right|}}_{\leq\left(2+K_{f}+D_{f}\right) \beta_{i}^{\prime}} \\
& \leq\left(9 K_{f}+15 D_{f}\right) \varepsilon+12\left(2+K_{f}+D_{f}\right) \beta(\varepsilon) .
\end{aligned}
$$

The last inequality follows from (4.3). As this bound holds for arbitrary $\varepsilon \in$ $(0,1 / 10)$ and $j \geq j_{0}(\varepsilon)$, this particularly proves $(4.9)$.

5. Approximation via Glivenko-Cantelli. In this section, we aim to apply a multivariate Glivenko-Cantelli theorem in order to approximate the empirical measure using the theoretical measure. Recall that a Glivenko-Cantelli theorem compares the empirical measure of a normalized sum of independent and identically distributed random variables with their distribution. At the end of this section, we will apply the following Glivenko-Cantelli theorem which was proved in [12] based on results by DeHardt and Wright; see $[3,17]$. Monotone functions on $\mathbb{R}^{k}$ were defined in (A4).

THEOREM 5.1. Let $(\Omega, \mathcal{A}, \mathbb{P})$ be a probability space and $X_{t}: \Omega \rightarrow \mathbb{R}^{k}$, $t \in \mathbb{N}$, independent and identically distributed random variables such that the distribution $\mu:=\mathbb{P}(X \in \cdot)$ is absolutely continuous with respect to a product measure $\bigotimes_{\ell=1}^{k} \mu_{\ell}$ on $\mathbb{R}^{k}$, where $\mu_{\ell}, \ell \in\{1, \ldots, k\}$, are $\sigma$-finite measures on $\mathbb{R}$. For each $n \in \mathbb{N}$, we denote by $L_{n}^{(\omega)}:=\frac{1}{n} \sum_{t=1}^{n} \delta_{X_{t}}$ the empirical distribution of $\left(X_{t}\right)_{t \in\{1, \ldots, n\}}$. Further, fix $M \in \mathbb{R}$ and let $\mathcal{M}:=\left\{g: \mathbb{R}^{k} \rightarrow \mathbb{R} \mid\right.$ $g$ is monotone, and $\left.\sup _{x \in \mathbb{R}^{k}}|g(x)| \leq M\right\}$.

Then, for all $\kappa>0$, there are $a=a(\kappa, M)>0$ and $b=b(\kappa, M)>0$ such that for all $n \in \mathbb{N}$, there exists an event $\Omega_{\kappa, n, M} \in \mathcal{A}$ with large probability $\mathbb{P}\left(\Omega_{\kappa, n, M}\right) \geq$ $1-b \exp (-a n)$, such that for all $\omega \in \Omega_{\kappa, n, M}$, we have

$$
\sup _{g \in \mathcal{M}}\left|\left\langle g, L_{n}^{(\omega)}-\mu\right\rangle\right| \leq \kappa .
$$

In particular, there exists a set $\Omega_{0} \in \mathcal{A}$ with $\mathbb{P}\left(\Omega_{0}\right)=1$ and $\sup _{g \in \mathcal{M}} \mid\langle g$, $\left.L_{n}^{(\omega)}-\mu\right\rangle \mid \stackrel{n \rightarrow \infty}{\longrightarrow} 0$ for all $\omega \in \Omega_{0}$.

In the present situation, we encounter several challenges when applying Theorem 5.1, caused by our tiling scheme: 
- Each $\Lambda_{j}$ is tiled using $N(\varepsilon)$ different shapes. Thus, the corresponding random variables (for different shapes) are not identically distributed.

- In an $\varepsilon$-quasi tiling, translates of the same shape $K_{i}$ are allowed to overlap. Thus, the corresponding random variables are not necessarily independent.

The first point can be handled by applying Glivenko-Cantelli theory for each shape $K_{i}$ separately. The second point is more challenging. The core of the following approach is the "generation of independence" by resampling of the overlapping areas using conditional probabilities and controlling errors introduced on the altered areas with their volume. Let us explain this in detail.

Fix $\varepsilon>0, i \in\{1, \ldots, N(\varepsilon)\}$ and $j \in \mathbb{N}, j \geq j_{0}(\varepsilon)$ (cf. Theorem 3.2), and consider Figure 1, which sketches a tile $K=K_{i}$, a finite set $\Lambda=\Lambda_{j}$, and the translations $K t, t \in T:=T_{i}^{j}(\varepsilon)$, of $K=K_{i}$ from an $\varepsilon$-quasi tiling. The sets

$$
U^{i, j, t}:=\left(K_{i}^{r} t\right) \backslash\left(K_{i}\left(T_{i}^{j}(\varepsilon) \backslash\{t\}\right)\right) \subseteq G, \quad t \in T,
$$

are marked with stripes. Their distance is at least

$$
d\left(U^{i, j, t}, U^{i, j, t^{\prime}}\right) \geq d\left(K_{i}^{r} t, G \backslash K_{i} t\right)>r, \quad t \neq t^{\prime},
$$

so the colors there are $\mathbb{P}$-independent from each other. Unfortunately, if we take only the values on $U^{i, j, t}, t \in T$, we will end up with an independent, but not identically distributed sample. We therefore resample independent colors in $K^{r} \backslash U^{i, j, t}$.
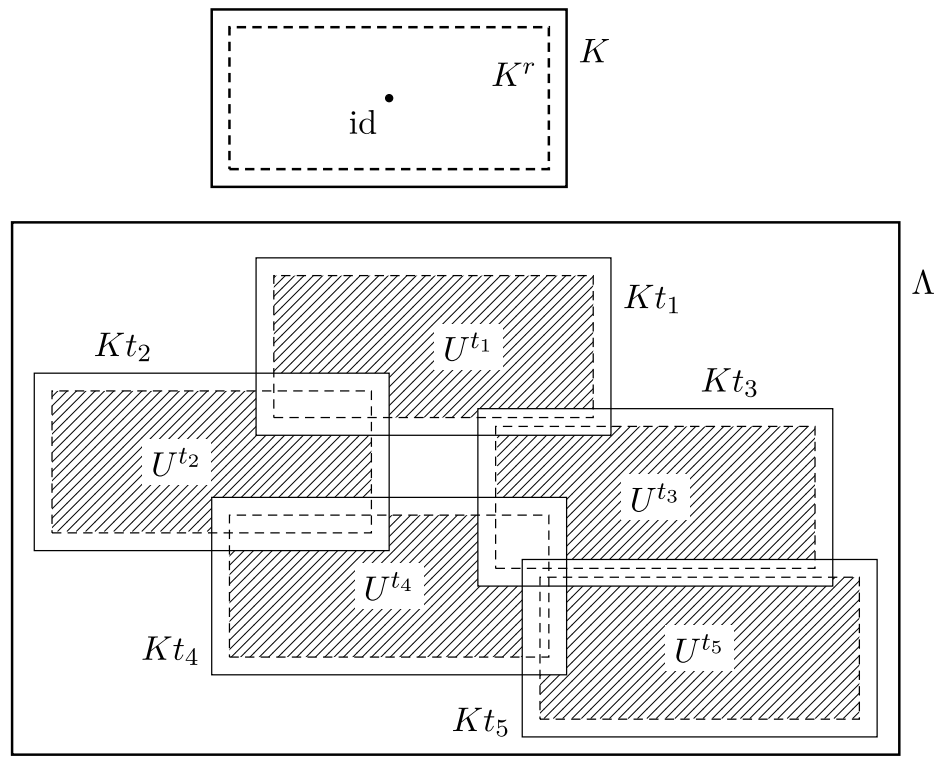

FIG. 1. $\varepsilon$-covering and independence structure: The set $\Lambda=\Lambda_{j} \subseteq G$ is $\varepsilon$-quasi covered by copies of $K=K_{i}$ with centers in $T=T_{i}^{j}(\varepsilon)=\left\{t_{1}, \ldots, t_{5}\right\}$. The sets $U^{t}=U^{i, j, t}, t \in T$, here marked by diagonal stripes, have at least distance $r$ and satisfy $\left|U^{t}\right| \geq(1-\varepsilon)|K|$. 
Fortunately, the sets $U^{i, j, t}$ are large enough to compensate this small random perturbation. The following lemma specifies the resampling procedure.

LEMMA 5.2. Let $\varepsilon>0$ and $I:=\bigcup_{i=1}^{N(\varepsilon)} \bigcup_{j=j_{0}(\varepsilon)}^{\infty}\{(i, j)\} \times T_{i}^{j}(\varepsilon)$. There exists a probability space $(\underline{\Omega}, \mathcal{B}(\underline{\Omega}), \underline{\mathbb{P}})$ and random variables $X, X^{i, j, t}: \underline{\Omega} \rightarrow \Omega$, $(i, j, t) \in I$, such that for all $(i, j, t) \in I$ :

(i) $X$ and $X^{i, j, t}$ have distribution $\mathbb{P}$,

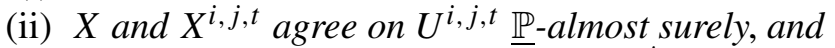

(iii) the random variables in the set $\left\{X^{i, j, t^{\prime}}\right\}_{t^{\prime} \in T_{i}^{j}(\varepsilon)}$ are $\underline{\mathbb{P}}$-independent.

ProOF. Theorem A.1 solves the problem of resampling in an abstract setting. We apply the result here as follows. Since we use the canonical probability space in our construction, we apply Theorem A.1 with $(S, \mathcal{S}):=(\Omega, \mathcal{A})$, $X:=\operatorname{id}_{\Omega}, I:=\bigcup_{i=1}^{N(\varepsilon)} \bigcup_{j=j_{0}(\varepsilon)}^{\infty}\{(i, j)\} \times T_{i}^{j}(\varepsilon)$, and $\mathcal{Y}_{j^{\prime}}:=\sigma\left(\Pi_{U^{j^{\prime}}}\right), j^{\prime} \in I$. Theorem A.1 provides the following quantities, which we here want to use as $(\underline{\Omega}, \underline{\mathcal{A}}, \underline{\mathbb{P}}):=(\underline{\Omega}, \underline{\mathcal{A}}, \underline{\mathbb{P}}), X:=X_{0}$, and $X^{i, j, t}:=X_{j^{\prime}}$ for all $j^{\prime}=(i, j, t) \in I$. The properties (i) and (ii) follow directly from Theorem A.1(i), (ii). With (5.2), Theorem A.1(iv) implies (iii).

Next, we control the error we introduce by using our independent samples instead of the dependent ones.

LEMMA 5.3. Fix $\varepsilon>0$, an admissible $f$ and $U \subseteq K \in \mathcal{F}$. For $\omega, \tilde{\omega} \in \Omega$ with $\omega_{U}=\tilde{\omega}_{U}$, we have

$$
\|f(\omega, K)-f(\tilde{\omega}, K)\| \leq 2 b(K)+2\left(2 D_{f}+K_{f}\right)|K \backslash U| .
$$

In particular, in the notation from (4.4)-(4.8) and with the corresponding empirical measure

$$
\underline{L}_{i, j}^{r, \underline{\omega}}(\varepsilon):=\frac{1}{\left|T_{i}^{j}(\varepsilon)\right|} \sum_{t \in T_{i}^{j}(\varepsilon)} \delta_{\left(\tau_{t} X^{i, j, t}(\underline{\omega})\right)_{K_{i}(\varepsilon)}}(\underline{\omega} \in \underline{\Omega}),
$$

we have for $\underline{\mathbb{P}}$-almost all $\underline{\omega} \in \underline{\Omega}$ that

$$
\left\|\left\langle f_{i}^{r}(\varepsilon), L_{i, j}^{r, X(\underline{\omega})}(\varepsilon)-\underline{L}_{i, j}^{r, \underline{\omega}}(\varepsilon)\right\rangle\right\| \leq 2 b\left(K_{i}^{r}(\varepsilon)\right)+2\left(2 D_{f}+K_{f}\right) \varepsilon\left|K_{i}^{r}(\varepsilon)\right| .
$$

Proof. The values of $\omega$ on $U$ determine $f(\omega, K)$ up to

$$
\begin{aligned}
& \|f(\omega, K)-f(\omega, U)\| \\
& \quad \leq\|f(\omega, K)-f(\omega, U)-f(\omega, K \backslash U)\|+\|f(\omega, K \backslash U)\| \\
& \quad \leq b(U)+b(K \backslash U)+\|f(\omega, K \backslash U)\| \\
& \quad \leq b(U)+\left(D_{f}+K_{f}\right)|K \backslash U| .
\end{aligned}
$$


With the fourth point in (A3), we can continue this estimate with

$$
b(U) \leq b(K \backslash(K \backslash U)) \leq b(K)+b(K \backslash U) \leq b(K)+D_{f}|K \backslash U| .
$$

We now employ the triangle inequality to show the first claim: For $\omega, \tilde{\omega} \in \Omega$ with $\omega_{U}=\tilde{\omega}_{U}$, we have

$$
\begin{aligned}
& \|f(\omega, K)-f(\tilde{\omega}, K)\| \\
& \quad \leq\|f(\omega, K)-f(\omega, U)\|+\|f(\tilde{\omega}, U)-f(\tilde{\omega}, K)\| \\
& \quad \leq 2\left(b(K)+\left(2 D_{f}+K_{f}\right)|K \backslash U|\right) .
\end{aligned}
$$

This calculation allows us to change $\omega$ on $K \backslash U$ to the independent values provided by Lemma 5.2. To implement this, observe that for $\underline{\mathbb{P}}$-almost all $\underline{\omega} \in \underline{\Omega}$ and all $i \in\{1, \ldots, N(\varepsilon)\}, j \in \mathbb{N}, j \geq j_{0}(\varepsilon)$ and $t \in T_{i}^{j}(\varepsilon)$, the set $U^{i, j, t}$ from (5.1) exhausts $K_{i}^{r}(\varepsilon) t$ up to a fraction of $\varepsilon:\left|K_{i}^{r}(\varepsilon) t \backslash U^{i, j, t}\right| \leq \varepsilon\left|K_{i}^{r}(\varepsilon)\right|$. By construction, on $U^{i, j, t}$, the colors are preserved: $U^{i, j, t} \subseteq\left\{g \in K_{i}^{r}(\varepsilon) t \mid X_{g}(\underline{\omega})=X_{g}^{i, j, t}(\underline{\omega})\right\}$. Together with Lemma 4.2 and the triangle inequality, this immediately implies for $\underline{\mathbb{P}}$-almost all $\underline{\omega} \in \underline{\Omega}$ that

$$
\begin{aligned}
& \left\|\left\langle f_{i}^{r}(\varepsilon), L_{i, j}^{r, X(\underline{\omega})}(\varepsilon)-\underline{L}_{i, j}^{r, \tilde{\omega}}(\varepsilon)\right\rangle\right\| \\
& \quad \leq \frac{1}{\left|T_{i}^{j}(\varepsilon)\right|} \sum_{t \in T_{i}^{j}(\varepsilon)}\left\|f\left(K_{i}^{r}(\varepsilon) t, \omega\right)-f\left(K_{i}^{r}(\varepsilon) t, X^{i, j, t}(\underline{\omega})\right)\right\| \\
& \quad \leq 2 b\left(K_{i}^{r}(\varepsilon)\right)+2\left(2 D_{f}+K_{f}\right) \varepsilon\left|K_{i}^{r}(\varepsilon)\right| .
\end{aligned}
$$
to

The empirical measure $L_{i, j}^{r, X(\underline{\omega})}$ formed by independent samples should converge

$$
\mathbb{P}_{i}^{r}(\varepsilon):=\mathbb{P}_{K_{i}^{r}(\varepsilon)}
$$

The following result makes this notion precise. It is the main result of this section.

Proposition 5.4. Let $G$ be a finitely generated amenable group, let $\mathcal{A} \in$ $\mathcal{B}(\mathbb{R})$ and $\left(\Omega:=\mathcal{A}^{G}, \mathcal{B}(\Omega), \mathbb{P}\right)$ a probability space such that $\mathbb{P}$ satisfies $(\mathrm{M} 1)$ to (M3). Moreover, let $\left(\Lambda_{n}\right)$ and $\left(Q_{n}\right)$ be Folner sequences, where $\left(Q_{n}\right)$ is nested and satisfies (4.1). For given $\varepsilon \in(0,1 / 10)$, let $K_{i}(\varepsilon), i \in\{1, \ldots, N(\varepsilon)\}$, and $j_{0}(\varepsilon)$ be given by Theorem 3.2. Furthermore, let $\mathcal{U}$ be an admissible set of admissible fields.

Then, for all $\kappa>0$, there exist $a\left(\varepsilon, \kappa, K_{\mathcal{U}}\right), b\left(\varepsilon, \kappa, K_{\mathcal{U}}\right)>0$ such that for all $j \geq j_{0}(\varepsilon)$, there is an event $\Omega_{j, \varepsilon, \kappa, K_{\mathcal{U}}} \in \mathcal{B}(\Omega)$ with large probability

$$
\mathbb{P}\left(\Omega_{j, \varepsilon, \kappa, K_{\mathcal{U}}}\right) \geq 1-b\left(\varepsilon, \kappa, K_{\mathcal{U}}\right) \exp \left(-a\left(\varepsilon, \kappa, K_{\mathcal{U}}\right)\left|\Lambda_{j}\right|\right)
$$


and the property that for all $\omega \in \Omega_{j, \varepsilon, \kappa, K_{\mathcal{U}}}$ and $f \in \mathcal{U}$, it holds true that

$$
\begin{aligned}
& \left\|\sum_{i=1}^{N(\varepsilon)} \eta_{i}(\varepsilon) \frac{\left\langle f_{i}^{r}(\varepsilon), L_{i, j}^{r, \omega}(\varepsilon)\right\rangle}{\left|K_{i}(\varepsilon)\right|}-\sum_{i=1}^{N(\varepsilon)} \eta_{i}(\varepsilon) \frac{\left\langle f_{i}^{r}(\varepsilon), \mathbb{P}_{i}^{r}(\varepsilon)\right\rangle}{\left|K_{i}(\varepsilon)\right|}\right\| \\
& \leq 2 \beta(\varepsilon)+2\left(2 D_{f}+K_{f}\right) \varepsilon+\kappa .
\end{aligned}
$$

In particular, there is an event $\tilde{\Omega} \in \mathcal{B}(\Omega)$ with $\mathbb{P}(\tilde{\Omega})=1$ such that for all $\omega \in \tilde{\Omega}$, we have

$$
\lim _{\varepsilon \searrow 0} \sup _{f \in \mathcal{U}}\left\|\sum_{i=1}^{N(\varepsilon)} \eta_{i}(\varepsilon) \frac{\left\langle f_{i}^{r}(\varepsilon), L_{i, j}^{r, \omega}(\varepsilon)\right\rangle}{\left|K_{i}(\varepsilon)\right|}-\sum_{i=1}^{N(\varepsilon)} \eta_{i}(\varepsilon) \frac{\left\langle f_{i}^{r}(\varepsilon), \mathbb{P}_{i}^{r}(\varepsilon)\right\rangle}{\left|K_{i}(\varepsilon)\right|}\right\|=0 .
$$

ProOF. Fix $f \in \mathcal{U}$. For $\varepsilon \in(0,1 / 10), j \in \mathbb{N}$ and $\omega \in \Omega$, two applications of the triangle inequality give

$$
\begin{aligned}
\Delta_{f}(\varepsilon, \omega) & :=\left\|\sum_{i=1}^{N(\varepsilon)} \eta_{i}(\varepsilon) \frac{\left\langle f_{i}^{r}(\varepsilon), L_{i, j}^{r, \omega}(\varepsilon)\right\rangle}{\left|K_{i}(\varepsilon)\right|}-\sum_{i=1}^{N(\varepsilon)} \eta_{i}(\varepsilon) \frac{\left\langle f_{i}^{r}(\varepsilon), \mathbb{P}_{i}^{r}(\varepsilon)\right\rangle}{\left|K_{i}(\varepsilon)\right|}\right\| \\
& \leq \sum_{i=1}^{N(\varepsilon)} \frac{\eta_{i}(\varepsilon)}{\left|K_{i}(\varepsilon)\right|}\left\|\left\langle f_{i}^{r}(\varepsilon), L_{i, j}^{r, \omega}(\varepsilon)-\mathbb{P}_{i}^{r}(\varepsilon)\right\rangle\right\| \\
& \leq \inf _{\underline{\omega} \in X^{-1}(\{\omega\})}\left(\sum_{i=1}^{N(\varepsilon)} \eta_{i}(\varepsilon) \gamma_{1}(i, j, \varepsilon, \underline{\omega})+\sum_{i=1}^{N(\varepsilon)} \eta_{i}(\varepsilon) \gamma_{2}(i, j, \varepsilon, \underline{\omega})\right),
\end{aligned}
$$

where $\underline{\omega} \in \underline{\Omega}$ extends $\omega$, that is, $X(\underline{\omega})=\omega$ in the notation of Lemma 5.2, and

$$
\begin{aligned}
\gamma_{1}(i, j, \varepsilon, \underline{\omega}) & :=\frac{\left\|\left\langle f_{i}^{r}(\varepsilon), L_{i, j}^{r, \omega}(\varepsilon)-\underline{L}_{i, j}^{r, \underline{\omega}}(\varepsilon)\right\rangle\right\|}{\left|K_{i}(\varepsilon)\right|} \text { and } \\
\gamma_{2}(i, j, \varepsilon, \underline{\omega}) & :=\frac{\left\|\left\langle f_{i}^{r}(\varepsilon), \underline{L}_{i, j}^{r, \underline{\omega}}(\varepsilon)-\mathbb{P}_{i}^{r}(\varepsilon)\right\rangle\right\|}{\left|K_{i}(\varepsilon)\right|} .
\end{aligned}
$$

By Lemma 5.3 and assumption (4.1), we see that for all $\underline{\omega} \in \underline{\Omega}$ with $X(\underline{\omega})=\omega$

$$
\gamma_{1}(i, j, \varepsilon, \underline{\omega}) \leq \frac{2 b\left(K_{i}^{r}(\varepsilon)\right)}{\left|K_{i}^{r}(\varepsilon)\right|}+2\left(2 D_{f}+K_{f}\right) \varepsilon \leq \frac{2 b\left(Q_{i}\right)}{\left|Q_{i}\right|}+2\left(2 D_{f}+K_{f}\right) \varepsilon .
$$

With Lemma 3.3(a) and (4.3), we yield the deterministic upper bound

$$
\sum_{i=1}^{N(\varepsilon)} \eta_{i}(\varepsilon) \gamma_{1}(i, j, \varepsilon, \underline{\omega}) \leq 2 \beta(\varepsilon)+2\left(2 D_{f}+K_{f}\right) \varepsilon
$$

for all $\underline{\omega} \in X^{-1}(\omega) \subseteq \underline{\Omega}$. By now, our overall inequality (5.3) reads

(5.4) $\Delta_{f}(\varepsilon, \omega) \leq 2 \beta(\varepsilon)+2\left(2 D_{f}+K_{f}\right) \varepsilon+\inf _{\underline{\omega} \in X^{-1}(\{\omega\})} \sum_{i=1}^{N(\varepsilon)} \eta_{i}(\varepsilon) \gamma_{2}(i, j, \varepsilon, \underline{\omega})$. 
To deal with $\gamma_{2}$, recall that the norm on the Banach space $\mathbb{B}$ our admissible fields map into is the sup-norm. We translate the sup-norm into the Glivenko-Cantelli setting as follows. Let

$$
\mathcal{M}_{f}:=\left\{g_{i, E}^{r}: \mathbb{R}^{\left|K_{i}^{r}(\varepsilon)\right|} \rightarrow \mathbb{R}, g_{i, E}^{r}(\omega):=f_{i}^{r}(\omega)(E) /\left|K_{i}(\varepsilon)\right| \mid E \in \mathbb{R}\right\} .
$$

Therefore, we can write

$$
\gamma_{2}(i, j, \varepsilon, \underline{\omega})=\sup _{g \in \mathcal{M}_{f}}\left|\left\langle g, \underline{L}_{i, j}^{r, \omega}(\varepsilon)-\mathbb{P}_{i}^{r}(\varepsilon)\right\rangle\right| \leq \sup _{f \in \mathcal{U}} \sup _{g \in \mathcal{M}_{f}}\left|\left\langle g, \underline{L}_{i, j}^{r, \omega}(\varepsilon)-\mathbb{P}_{i}^{r}(\varepsilon)\right\rangle\right| .
$$

From (2.5), we see that the fields in $\mathcal{M}_{\mathcal{U}}:=\bigcup_{f \in \mathcal{U}} \mathcal{M}_{f}$ are bounded by $K_{\mathcal{U}}$. As assumed in (A4), the fields in $\mathcal{M}_{\mathcal{U}}$ are also monotone. By Lemma 5.2(iii), the samples are independent, also. This is crucial in order to invoke Theorem 5.1. We thus obtain that, for each $\kappa>0, \varepsilon \in(0,1 / 10), i \in\{1, \ldots, N(\varepsilon)\}$ and $j \in \mathbb{N}$, $j \geq j_{0}(\varepsilon)$, there are $a_{i} \equiv a\left(i, \varepsilon, \kappa, K_{\mathcal{U}}\right)>0, b_{i} \equiv b\left(i, \varepsilon, \kappa, K_{\mathcal{U}}\right)>0$ and $\underline{\Omega}_{i, j} \equiv$ $\underline{\Omega}_{i, j, \varepsilon, \kappa, K_{\mathcal{U}}} \in \mathcal{B}(\underline{\Omega})$ such that

$$
\underline{\mathbb{P}}\left(\underline{\Omega}_{i, j}\right) \geq 1-b_{i} \exp \left(-a_{i}\left|T_{i}^{j}(\varepsilon)\right|\right) \quad \text { and } \quad \sup _{\underline{\omega} \in \underline{\Omega}_{i, j}} \gamma_{2}(i, j, \varepsilon, \underline{\omega}) \leq \kappa .
$$

We need this estimate for all $i \in\{1, \ldots, N(\varepsilon)\}$ simultaneously and consider

$$
\underline{\Omega}_{j} \equiv \underline{\Omega}_{j, \varepsilon, \kappa, K_{\mathcal{U}}}:=\bigcap_{i=1}^{N(\varepsilon)} \underline{\Omega}_{i, j} .
$$

To estimate the probability of $\underline{\Omega}_{j}$ is the next step. From (3.3) and Lemma 3.3(b), we note that

$$
\left|T_{i}^{j}(\varepsilon)\right| \geq\left(\eta_{i}(\varepsilon)-\frac{\varepsilon^{2}}{N(\varepsilon)}\right) \frac{\left|\Lambda_{j}\right|}{\left|K_{i}(\varepsilon)\right|} \geq \frac{(1-\varepsilon) \varepsilon}{N(\varepsilon)\left|K_{i}(\varepsilon)\right|}\left|\Lambda_{j}\right| .
$$

With the definition

$$
a \equiv a_{\varepsilon, \kappa, K_{\mathcal{U}}}:=\frac{(1-\varepsilon) \varepsilon}{N(\varepsilon)} \min _{i \in\{1, \ldots, N(\varepsilon)\}} \frac{a_{i}}{\left|K_{i}(\varepsilon)\right|} \quad \text { and } \quad b \equiv b_{\varepsilon, \kappa, K_{\mathcal{U}}}:=2 \sum_{i=1}^{N(\varepsilon)} b_{i},
$$

we get $\underline{\mathbb{P}}\left(\underline{\Omega}_{i, j}\right) \geq 1-b_{i} \exp \left(-a\left|\Lambda_{j}\right|\right)$ and

$$
\underline{\mathbb{P}}\left(\underline{\Omega}_{j}\right)=1-\mathbb{P}\left(\bigcup_{i=1}^{N(\varepsilon)} \underline{\Omega} \backslash \underline{\Omega}_{i, j}\right) \geq 1-\sum_{i=1}^{N(\varepsilon)} \mathbb{P}\left(\underline{\Omega} \backslash \underline{\Omega}_{i, j}\right) \geq 1-\frac{b \exp \left(-a\left|\Lambda_{j}\right|\right)}{2} .
$$

Next, we should transition from $(\underline{\Omega}, \mathcal{B}(\underline{\Omega}), \mathbb{P})$ to $(\Omega, \mathcal{B}(\Omega), \mathbb{P})$. The set $X\left(\underline{\Omega}_{j}\right) \subseteq$ $\Omega$ seems to be a good candidate, because for all $\omega \in X\left(\underline{\Omega}_{j}\right)$, there exists $\underline{\omega} \in$ $X^{-1}(\{\omega\}) \cap \bigcap_{i=1}^{N(\varepsilon)} \underline{\Omega}_{i, j}$, and thus we can estimate

$$
\inf _{\underline{\omega} \in X^{-1}(\{\omega\})} \sum_{i=1}^{N(\varepsilon)} \eta_{i}(\varepsilon) \gamma_{2}(i, j, \varepsilon, \underline{\omega}) \leq \sum_{i=1}^{N(\varepsilon)} \eta_{i}(\varepsilon) \kappa \leq \kappa .
$$


Together with (5.4), this inequality shows the claimed bound on $\Delta_{f}(\varepsilon, \omega)$ for all $\omega \in X\left(\underline{\Omega}_{j}\right)$.

Unfortunately, the image of a measurable set under a measurable map is not necessarily measurable, but only analytic; see [2], Theorem 10.23. At least the outer measure of our candidate is bounded from below by

$$
\begin{aligned}
\mathbb{P}^{*}\left(X\left(\underline{\Omega}_{j}\right)\right) & :=\inf _{B \in \mathcal{B}(\Omega), X\left(\underline{\Omega}_{j}\right) \subseteq B} \mathbb{P}(B) \\
& =\inf _{B \in \mathcal{B}(\Omega), X\left(\underline{\Omega}_{j}\right) \subseteq B} \mathbb{P}(X \in B) \\
& \geq \inf _{B \in \mathcal{B}(\Omega), X\left(\underline{\Omega}_{j}\right) \subseteq B} \mathbb{P}\left(\underline{\Omega}_{j}\right) \\
& =\underline{\mathbb{P}}\left(\underline{\Omega}_{j}\right) \geq 1-b \exp \left(-a\left|\Lambda_{j}\right|\right) / 2 .
\end{aligned}
$$

From [2], Lemma 10.36, we learn that $\mathbb{P}^{*}$ is a nice capacity, and the Choquet capacity theorem [2], Theorem 10.39 , states for the analytic set $X\left(\underline{\Omega}_{j}\right)$ that

$$
\mathbb{P}^{*}\left(X\left(\underline{\Omega}_{j}\right)\right)=\sup _{K \subseteq X\left(\underline{\Omega}_{j}\right) \text { compact }} \mathbb{P}(K) .
$$

Thus, there exists a compact subset $\Omega_{j, \varepsilon, \kappa, K_{\mathcal{U}}} \subseteq X\left(\underline{\Omega}_{j}\right)$ with probability at least $1-b \exp \left(-a\left|\Lambda_{j}\right|\right)$.

We complete the proof with a standard Borel-Cantelli argument to show that $\tilde{\Omega}$ exists as claimed. For all $\kappa>0$, the events

$$
A_{\kappa}:=\bigcup_{n=j_{0}(\varepsilon)}^{\infty} \bigcap_{j=n}^{\infty} \Omega_{j, \varepsilon, \kappa, K_{\mathcal{U}}}
$$

have probability 1 , since

$$
\sum_{j=j_{0}(\varepsilon)}^{\infty} \mathbb{P}\left(\Omega \backslash \Omega_{j, \varepsilon, \kappa, K_{\mathcal{U}}}\right) \leq \sum_{j=j_{0}(\varepsilon)}^{\infty} b \exp \left(-a\left|\Lambda_{j}\right|\right) \leq b \sum_{j=j_{0}(\varepsilon)}^{\infty} \exp (-a)^{j}<\infty .
$$

Note that by (5.4), $\beta(\varepsilon) \rightarrow 0$ and by construction of $A_{k}$, for all $\omega \in A_{\kappa}$, we have

$$
\lim _{\varepsilon \searrow 0} \sup _{f \in \mathcal{U}} \Delta_{f}(\varepsilon, \omega) \leq \kappa
$$

Thus, the event $\tilde{\Omega}:=\bigcap_{k \in \mathbb{N}} A_{1 / k}$ has full probability $\mathbb{P}(\tilde{\Omega})=1$, and for all $\omega \in \tilde{\Omega}$, we have $\lim _{\mathcal{E} \searrow 0} \sup _{f \in \mathcal{U}} \Delta_{f}(\varepsilon, \omega)=0$.

6. Almost additivity and Cauchy sequences. The following calculations are devoted to a Cauchy sequence argument to obtain the desired limit function $f^{*}$. 
LEMMA 6.1. Let $G$ be a finitely generated amenable group, let $\mathcal{A} \in \mathcal{B}(\mathbb{R})$ and $\left(\Omega=\mathcal{A}^{G}, \mathcal{B}(\Omega), \mathbb{P}\right)$ a probability space such that $\mathbb{P}$ satisfies $(\mathrm{M} 1)$ to $(\mathrm{M} 3)$. Moreover, let $f$ be an admissible field and $\left(Q_{n}\right)$ a nested Følner sequence satisfying (4.1). Then there exists $f^{*} \in \mathbb{B}$ with

$$
\lim _{\varepsilon \searrow 0}\left\|\sum_{i=1}^{N(\varepsilon)} \eta_{i}(\varepsilon) \frac{\left\langle f_{i}^{r}(\varepsilon), \mathbb{P}_{i}^{r}(\varepsilon)\right\rangle}{\left|K_{i}(\varepsilon)\right|}-f^{*}\right\|=0,
$$

where for $k \in \mathbb{N}$ and $\varepsilon \in(1 /(k+1), 1 / k)$ the sets $K_{i}(\varepsilon), i \in\{1, \ldots, N(\varepsilon)\}$ are extracted from the sequence $\left(Q_{n+k}\right)_{n}$ via Theorem 3.2. The approximation error is bounded by

$$
\left\|\sum_{j=1}^{N(\varepsilon)} \eta_{j}(\varepsilon) \frac{\left\langle f_{j}^{r}(\varepsilon), \mathbb{P}_{j}^{r}(\varepsilon)\right\rangle}{\left|K_{j}(\varepsilon)\right|}-f^{*}\right\| \leq\left(9 K_{f}+11 D_{f}\right) \varepsilon+5\left(4+K_{f}+D_{f}\right) \beta(\varepsilon) .
$$

PROOF. In order to prove the existence of $f^{*}$, we study for $\varepsilon, \delta \in(0,1 / 10)$ the difference

$$
\mathcal{D}(\varepsilon, \delta):=\left\|\sum_{j=1}^{N(\varepsilon)} \eta_{j}(\varepsilon) \frac{\left\langle f_{j}^{r}(\varepsilon), \mathbb{P}_{j}^{r}(\varepsilon)\right\rangle}{\left|K_{j}(\varepsilon)\right|}-\sum_{i=1}^{N(\delta)} \eta_{i}(\delta) \frac{\left\langle f_{i}^{r}(\delta), \mathbb{P}_{i}^{r}(\delta)\right\rangle}{\left|K_{i}(\delta)\right|}\right\| .
$$

Our aim is to show $\lim _{\delta \searrow 0} \lim _{\varepsilon \searrow 0} \mathcal{D}(\varepsilon, \delta)=0$. To prove this, we insert terms which interpolate between the minuend and the subtrahend. These terms will be given using Theorem 3.2. For each $\varepsilon \in(1 /(k+1), 1 / k]$, we apply Theorem 3.2 to choose the sets $K_{j}(\varepsilon), j=1, \ldots, N(\varepsilon)$, from the Følner sequence $\left(Q_{n+k}\right)_{n \in \mathbb{N}}$. The particular choice of the sets $K_{j}(\varepsilon), j=1, \ldots, N(\varepsilon)$, as elements of the sequence $\left(Q_{n+k}\right)_{n}$ ensures that for given $\delta>0$ we find $\varepsilon_{0}>0$ such that for arbitrary $\varepsilon \in\left(0, \varepsilon_{0}\right)$ each $K_{j}(\varepsilon), j=1, \ldots, N(\varepsilon)$, can be $\delta$-quasi tiled with the elements $K_{i}(\delta), i=1, \ldots, N(\delta)$. As in Theorem 3.2, we denote the associated center sets by $T_{i}^{j}(\delta)$, where we emphasize the dependence on the parameter $\delta$.

For $K \in \mathcal{F}$, we use the notation

$$
F(K):=\left\langle f_{K}, \mathbb{P}_{K}\right\rangle
$$

and hence for the tiles $K_{j}(\varepsilon), i=1, \ldots, N(\varepsilon)$, we write $F\left(K_{i}^{r}(\varepsilon)\right):=\left\langle f_{i}^{r}(\varepsilon)\right.$, $\left.\mathbb{P}_{i}^{r}(\varepsilon)\right\rangle$. The function $F$ is translation invariant, that is, for all $K \in \mathcal{F}$ and $t \in G$ we have $F(K t)=F(K)$.

With the convention (6.1) and using the triangle inequality, we obtain $\mathcal{D}(\varepsilon, \delta) \leq$ $\mathcal{D}_{1}(\varepsilon, \delta)+\mathcal{D}_{2}(\varepsilon, \delta)$, where

$$
\begin{aligned}
\mathcal{D}_{1}(\varepsilon, \delta) & :=\left\|\sum_{j=1}^{N(\varepsilon)} \eta_{j}(\varepsilon) \frac{F\left(K_{j}^{r}(\varepsilon)\right)-\sum_{i=1}^{N(\delta)}\left|T_{i}^{j}(\delta)\right| F\left(K_{i}^{r}(\delta)\right)}{\left|K_{j}(\varepsilon)\right|}\right\|, \quad \text { and } \\
\mathcal{D}_{2}(\varepsilon, \delta) & :=\left\|\sum_{j=1}^{N(\varepsilon)} \eta_{j}(\varepsilon) \frac{\sum_{i=1}^{N(\delta)}\left|T_{i}^{j}(\delta)\right| F\left(K_{i}^{r}(\delta)\right)}{\left|K_{j}(\varepsilon)\right|}-\sum_{i=1}^{N(\delta)} \eta_{i}(\delta) \frac{F\left(K_{i}^{r}(\delta)\right)}{\left|K_{i}(\delta)\right|}\right\| .
\end{aligned}
$$


The translation invariance of $F$ and the triangle inequality yield

$$
\mathcal{D}_{1}(\varepsilon, \delta) \leq \sum_{j=1}^{N(\varepsilon)} \frac{\eta_{j}(\varepsilon)}{\left|K_{j}(\varepsilon)\right|}\left\|F\left(K_{j}^{r}(\varepsilon)\right)-\sum_{i=1}^{N(\delta)} \sum_{t \in T_{i}^{j}(\delta)} F\left(K_{i}^{r}(\delta) t\right)\right\| .
$$

We decompose $K_{j}^{r}(\varepsilon)$ in the following way:

$$
\begin{aligned}
K_{j}^{r}(\varepsilon)= & \bigcup_{i=1}^{N(\delta)} \bigcup_{t \in T_{i}^{j}(\delta)} K_{i}^{r}(\delta) t \dot{\cup} K_{j}^{r}(\varepsilon) \backslash \bigcup_{i=1}^{N(\delta)} K_{i}(\delta) T_{i}^{j}(\delta) \\
& \dot{\cup}\left(\left(K_{j}^{r}(\varepsilon) \backslash \bigcup_{i=1}^{N(\delta)} K_{i}^{r}(\delta) T_{i}^{j}(\delta)\right) \cap \bigcup_{i=1}^{N(\delta)}\left(K_{i}(\delta) \cap \partial^{r}\left(K_{i}(\delta)\right)\right) T_{i}^{j}(\delta)\right) \\
= & \alpha_{1} \dot{\cup} \alpha_{2} \dot{\cup} \alpha_{3} .
\end{aligned}
$$

By definition of the function $F$, the almost additivity of the admissible field $f$ inherits to $F$. Note that $\delta$-disjointness of the sets $K_{i} t, t \in T_{i}^{j}(\delta)$ implies $\delta$ disjointness of the sets $K_{i}^{r} t, t \in T_{i}^{j}(\delta)$. Therefore, applying almost additivity, Lemma 3.5 and the properties of admissible fields and the boundary term we obtain

$$
\begin{aligned}
&\left\|F\left(K_{j}^{r}(\varepsilon)\right)-\sum_{i=1}^{N(\delta)} \sum_{t \in T_{i}^{j}(\delta)} F\left(K_{i}^{r}(\delta) t\right)\right\| \\
& \leq\left\|F\left(K_{j}^{r}(\varepsilon)\right)-\sum_{i=1}^{3} F\left(\alpha_{i}\right)\right\|+\left\|F\left(\alpha_{1}\right)-\sum_{i=1}^{N(\delta)} \sum_{t \in T_{i}^{j}(\delta)} F\left(K_{i}^{r}(\delta)\right)\right\| \\
& \quad+\left\|F\left(\alpha_{2}\right)\right\|+\left\|F\left(\alpha_{3}\right)\right\| \\
& \leq \sum_{i=1}^{3} b\left(\alpha_{i}\right)+\delta\left(3 K_{f}+9 D_{f}\right)\left|K_{j}(\varepsilon)\right| \\
& \quad+3 \sum_{i=1}^{N(\delta)} \sum_{t \in T_{i}^{j}} b\left(K_{i}^{r}(\delta)\right)+K_{f}\left|\alpha_{2}\right|+K_{f}\left|\alpha_{3}\right| \\
& \leq \delta\left(3 K_{f}+9 D_{f}\right)\left|K_{j}(\varepsilon)\right| \\
&+4 \sum_{i=1}^{N(\delta)} \sum_{t \in T_{i}^{j}(\delta)} b\left(K_{i}^{r}(\delta)\right)+\left(K_{f}+D_{f}\right)\left|\alpha_{2}\right|+\left(K_{f}+D_{f}\right)\left|\alpha_{3}\right| .
\end{aligned}
$$

Next, we estimate the sizes of $\alpha_{2}$ and $\alpha_{3}$. For $\alpha_{3}$, we drop some of the intersections in its definition. In order to give a bound on the size of $\alpha_{2}$, we use that $K_{j}^{r}(\varepsilon)$ is 
$(1-2 \varepsilon)$-covered by $\left\{K_{i}^{r}(\delta) \mid i\right\}$, more specifically, part (iii) in Definition 3.1. We obtain

$$
\left|\alpha_{2}\right| \leq 2 \delta\left|K_{j}(\varepsilon)\right| \quad \text { and } \quad\left|\alpha_{3}\right| \leq \sum_{i=1}^{N(\delta)}\left|T_{i}^{j}(\delta)\right|\left|\partial^{r}\left(K_{i}(\delta)\right)\right|,
$$

and therewith achieve

$$
\begin{aligned}
\| F\left(K_{j}^{r}(\varepsilon)\right) & -\sum_{i=1}^{N(\delta)} \sum_{t \in T_{i}^{j}(\delta)} F\left(K_{i}^{r}(\delta) t\right) \| \\
\leq & \delta\left(5 K_{f}+11 D_{f}\right)\left|K_{j}(\varepsilon)\right| \\
& +\sum_{i=1}^{N(\delta)}\left|T_{i}^{j}(\delta)\right|\left(4 b\left(K_{i}^{r}(\delta)\right)+\left(K_{f}+D_{f}\right)\left|\partial^{r}\left(K_{i}(\delta)\right)\right|\right) .
\end{aligned}
$$

This together with (6.2) and part (a) of Lemma 3.3 yields

$$
\begin{aligned}
\mathcal{D}_{1}(\varepsilon, \delta) & \\
\leq & \sum_{j=1}^{N(\varepsilon)}\left(\delta\left(5 K_{f}+11 D_{f}\right) \eta_{j}(\varepsilon)\right. \\
& \left.+\sum_{i=1}^{N(\delta)} \frac{\eta_{j}(\varepsilon)\left|T_{i}^{j}(\delta)\right|}{\left|K_{j}(\varepsilon)\right|}\left(4 b\left(K_{i}^{r}(\delta)\right)+\left(K_{f}+D_{f}\right)\left|\partial^{r}\left(K_{i}(\delta)\right)\right|\right)\right) \\
\leq & \delta\left(5 K_{f}+11 D_{f}\right) \\
& +\sum_{j=1}^{N(\varepsilon)} \sum_{i=1}^{N(\delta)} \frac{\eta_{j}(\varepsilon)\left|T_{i}^{j}(\delta)\right|}{\left|K_{j}(\varepsilon)\right|}\left(4 b\left(K_{i}^{r}(\delta)\right)+\left(K_{f}+D_{f}\right)\left|\partial^{r}\left(K_{i}(\delta)\right)\right|\right) .
\end{aligned}
$$

As $\delta$ is assumed to be smaller than $1 / 10$, we can apply Corollary 3.4, which gives for arbitrary $i \in\{1, \ldots, N(\delta)\}$ and $j \in\{1, \ldots, N(\varepsilon)\}$

$$
\frac{\left|T_{i}^{j}(\delta)\right|}{\left|K_{j}(\varepsilon)\right|} \leq \frac{\eta_{i}(\delta)}{\left|K_{i}(\delta)\right|}+4 \frac{\delta \eta_{i}(\delta)}{\left|K_{i}(\delta)\right|} \leq 5 \frac{\eta_{i}(\delta)}{\left|K_{i}(\delta)\right|} .
$$

Inserting this in the last estimate for $\mathcal{D}_{1}(\varepsilon, \delta)$ implies together with part (a) of Lemma 3.3 that

$$
\begin{aligned}
\mathcal{D}_{1}(\varepsilon, \delta) \leq & \delta\left(5 K_{f}+11 D_{f}\right) \\
& +\sum_{i=1}^{N(\delta)} \frac{5 \eta_{i}(\delta)}{\left|K_{i}(\delta)\right|}\left(4 b\left(K_{i}^{r}(\delta)\right)+\left(K_{f}+D_{f}\right)\left|\partial^{r}\left(K_{i}(\delta)\right)\right|\right) .
\end{aligned}
$$


Now, we use the monotonicity assumption in (4.1), which allows to replace the elements $K_{i}^{r}(\delta)$ and $K_{i}(\delta)$ by $Q_{i}^{r}$ and $Q_{i}$, respectively,

$$
\begin{aligned}
\mathcal{D}_{1}(\varepsilon, \delta) \leq & \delta\left(5 K_{f}+11 D_{f}\right) \\
& +\sum_{i=1}^{N(\delta)} \frac{5 \eta_{i}(\delta)}{\left|Q_{i}\right|}\left(4 b\left(Q_{i}^{r}\right)+\left(K_{f}+D_{f}\right)\left|\partial^{r}\left(Q_{i}\right)\right|\right) .
\end{aligned}
$$

Let us proceed with the estimation of $\mathcal{D}_{2}(\varepsilon, \delta)$ :

$$
\mathcal{D}_{2}(\varepsilon, \delta)=\left\|\sum_{i=1}^{N(\delta)} F\left(K_{i}^{r}(\delta)\right)\left(\sum_{j=1}^{N(\varepsilon)} \eta_{j}(\varepsilon) \frac{\left|T_{i}^{j}(\delta)\right|}{\left|K_{j}(\varepsilon)\right|}-\frac{\eta_{i}(\delta)}{\left|K_{i}(\delta)\right|}\right)\right\| .
$$

With the triangle inequality, Corollary 3.4 and part (a) of Lemma 3.3 we obtain

$$
\begin{aligned}
& \left|\sum_{j=1}^{N(\varepsilon)} \eta_{j}(\varepsilon) \frac{\left|T_{i}^{j}(\delta)\right|}{\left|K_{j}(\varepsilon)\right|}-\frac{\eta_{i}(\delta)}{\left|K_{i}(\delta)\right|}\right| \\
& \quad \leq \sum_{j=1}^{N(\varepsilon)} \eta_{j}(\varepsilon)\left|\frac{\left|T_{i}^{j}(\delta)\right|}{\left|K_{j}(\varepsilon)\right|}-\frac{\eta_{i}(\delta)}{\left|K_{i}(\delta)\right|}\right|+\left|\sum_{j=1}^{N(\varepsilon)} \eta_{j}(\varepsilon)-1\right| \frac{\eta_{i}(\delta)}{\left|K_{i}(\delta)\right|} \\
& \quad \leq \sum_{j=1}^{N(\varepsilon)} \eta_{j}(\varepsilon) \frac{4 \delta \eta_{i}(\delta)}{\left|K_{i}(\delta)\right|}+\frac{\varepsilon \eta_{i}(\delta)}{\left|K_{i}(\delta)\right|} \leq \frac{4 \delta \eta_{i}(\delta)}{\left|K_{i}(\delta)\right|}+\frac{\varepsilon \eta_{i}(\delta)}{\left|K_{i}(\delta)\right|} .
\end{aligned}
$$

This together with (6.4) gives the bound

$$
\mathcal{D}_{2}(\varepsilon, \delta) \leq \sum_{i=1}^{N(\delta)} K_{f}\left|K_{i}^{r}(\delta)\right|\left(\frac{4 \delta \eta_{i}(\delta)}{\left|K_{i}(\delta)\right|}+\frac{\varepsilon \eta_{i}(\delta)}{\left|K_{i}(\delta)\right|}\right) \leq 4 K_{f} \delta+K_{f} \varepsilon .
$$

Thus, the estimates of $\mathcal{D}_{1}(\varepsilon, \delta)$ and $\mathcal{D}_{2}(\varepsilon, \delta)$ in (6.3) and (6.5) together yield

$$
\mathcal{D}(\varepsilon, \delta) \leq K_{f} \varepsilon+\delta\left(9 K_{f}+11 D_{f}\right)
$$

$$
+\sum_{i=1}^{N(\delta)} \frac{5 \eta_{i}(\delta)}{\left|Q_{i}\right|}\left(4 b\left(Q_{i}^{r}\right)+\left(K_{f}+D_{f}\right)\left|\partial^{r}\left(Q_{i}\right)\right|\right)
$$

for all $\delta>0$ and $\varepsilon \in\left(0, \varepsilon_{0}(\delta)\right)$. Applying part (c) of Lemma 3.3, we see

$$
\lim _{\delta \searrow 0 \varepsilon \searrow 0} \lim _{\varepsilon} \mathcal{D}(\varepsilon, \delta)=0 .
$$

Using a Cauchy argument and the fact that $\mathbb{B}$ is a Banach space, we obtain that there exists an element $f^{*} \in \mathbb{B}$ with

$$
\lim _{\varepsilon \searrow 0}\left\|\sum_{j=1}^{N(\varepsilon)} \eta_{j}(\varepsilon) \frac{\left\langle f_{j}^{r}(\varepsilon), \mathbb{P}_{j}^{r}(\varepsilon)\right\rangle}{\left|K_{j}(\varepsilon)\right|}-f^{*}\right\|=0 .
$$


In order to get the error estimate for finite $\delta>0$, we use (6.6), Lemma 3.3(c) and (4.1) as follows:

$$
\begin{aligned}
& \left\|\sum_{j=1}^{N(\delta)} \eta_{j}(\delta) \frac{\left\langle f_{j}^{r}(\delta), \mathbb{P}_{j}^{r}(\delta)\right\rangle}{\left|K_{j}(\delta)\right|}-f^{*}\right\| \\
& \quad=\lim _{\varepsilon \searrow 0} \mathcal{D}(\varepsilon, \delta) \\
& \quad \leq\left(9 K_{f}+11 D_{f}\right) \delta+\sum_{i=1}^{N(\delta)} \frac{5 \eta_{i}(\delta)}{\left|Q_{i}\right|}\left(4 b\left(Q_{i}^{r}\right)+\left(K_{f}+D_{f}\right)\left|\partial^{r}\left(Q_{i}\right)\right|\right) \\
& \quad \leq\left(9 K_{f}+11 D_{f}\right) \delta+5\left(4+K_{f}+D_{f}\right) \beta(\delta) .
\end{aligned}
$$

7. Proof of the main theorem. We will prove a slightly more explicit statement which tracks the geometric error in terms of $\varepsilon$ and the probabilistic error in terms of $\kappa$ separately. Theorem 2.5 is implied by the choice $\kappa:=\sqrt{\varepsilon}$. Recall that $\mathbb{B}$ is the Banach space of bounded and right-continuous functions from $\mathbb{R}$ to $\mathbb{R}$.

THEOREM 7.1. Let $G$ be a finitely generated amenable group. Further, let $\mathcal{A} \in \mathcal{B}(\mathbb{R})$ and $\left(\Omega=\mathcal{A}^{G}, \mathcal{B}(\Omega), \mathbb{P}\right)$ a probability space such that $\mathbb{P}$ satisfies $(\mathrm{M} 1)$ to (M3). Finally, let $\mathcal{U}$ be an admissible set of admissible fields with common bound $K_{\mathcal{U}} ; c f$. Definition 2.2.

Then there exists a limit element $f^{*} \in \mathbb{B}$ with the following properties. For each Folner sequence $\left(\Lambda_{n}\right), \varepsilon \in(0,1 / 10)$ and $\kappa>0$, there exist $j_{0}(\varepsilon) \in \mathbb{N}$, which is independent of $\kappa$ and $K_{\mathcal{U}}$, and $a\left(\varepsilon, \kappa, K_{\mathcal{U}}\right), b\left(\varepsilon, \kappa, K_{\mathcal{U}}\right)>0$, such that for all $j \in$ $\mathbb{N}, j \geq j_{0}(\varepsilon)$, there is an event $\Omega_{j, \varepsilon, \kappa, K_{\mathcal{U}}} \in \mathcal{B}(\Omega)$ with the properties

$$
\mathbb{P}\left(\Omega_{j, \varepsilon, \kappa, K_{\mathcal{U}}}\right) \geq 1-b\left(\varepsilon, \kappa, K_{\mathcal{U}}\right) \exp \left(-a\left(\varepsilon, \kappa, K_{\mathcal{U}}\right)\left|\Lambda_{j}\right|\right)
$$

and

$$
\begin{aligned}
& \left\|\frac{f\left(\Lambda_{j}, \omega\right)}{\left|\Lambda_{j}\right|}-f^{*}\right\| \\
& \quad \leq\left(37 K_{f}+47 D_{f}+46\right) \sqrt{\varepsilon}+\kappa \quad \text { for all } \omega \in \Omega_{j, \varepsilon, \kappa, K_{\mathcal{U}}} \text { and all } f \in \mathcal{U} .
\end{aligned}
$$

PROOF. We follow the path prescribed in the previous chapters and:

- quasi tile $\Lambda_{j}, j \geq j_{0}(\varepsilon)$, with $K_{i}(\varepsilon), i=1, \ldots, N(\varepsilon)$ (see Theorem 3.2),

- approximate $\left|\Lambda_{j}\right|^{-1} f\left(\Lambda_{j}, \omega\right)$ with the empirical measures $L_{i, j}^{r, \omega}(\varepsilon)$; cf. (4.8) and Lemma 4.3,

- express the empirical measures by their limiting counterparts $\mathbb{P}_{i}^{r}(\varepsilon)$ with Lemma 5.4, and

- use the Cauchy property of the remaining terms to obtain a limiting function $f^{*}$; see Lemma 6.1. 
To confirm the error estimate, we employ the triangle inequality

$$
\begin{aligned}
& \left\|\frac{f\left(\Lambda_{j}, \omega\right)}{\left|\Lambda_{j}\right|}-f^{*}\right\| \\
& \leq\left\|\frac{f\left(\Lambda_{j}, \omega\right)}{\left|\Lambda_{j}\right|}-\sum_{i=1}^{N(\varepsilon)} \eta_{i}(\varepsilon) \frac{\left\langle f_{i}^{r}(\varepsilon), L_{i, j}^{r, \omega}(\varepsilon)\right\rangle}{\left|K_{i}(\varepsilon)\right|}\right\| \\
& \quad+\left\|\sum_{i=1}^{N(\varepsilon)} \eta_{i}(\varepsilon) \frac{\left\langle f_{i}^{r}(\varepsilon), L_{i, j}^{r, \omega}(\varepsilon)\right\rangle}{\left|K_{i}(\varepsilon)\right|}-\sum_{i=1}^{N(\varepsilon)} \eta_{i}(\varepsilon) \frac{\left\langle f_{i}^{r}(\varepsilon), \mathbb{P}_{i}^{r}(\varepsilon)\right\rangle}{\left|K_{i}(\varepsilon)\right|}\right\| \\
& \quad+\left\|\sum_{i=1}^{N(\varepsilon)} \eta_{i}(\varepsilon) \frac{\left\langle f_{i}^{r}(\varepsilon), \mathbb{P}_{i}^{r}(\varepsilon)\right\rangle}{\left|K_{i}(\varepsilon)\right|}-f^{*}\right\|=: \Delta(\varepsilon, j, \omega) .
\end{aligned}
$$

By Lemmas 6.1 and 4.3 and Proposition 5.4, we immediately get that there is an event $\tilde{\Omega} \in \mathcal{B}(\Omega)$ with full probability $\mathbb{P}(\tilde{\Omega})=1$ such that $\lim _{\mathcal{E} \backslash 0} \lim _{j \rightarrow \infty} \Delta(\varepsilon$, $j, \omega)=0$ for all $\omega \in \tilde{\Omega}$. Furthermore, Lemma 5.4 provides the event $\Omega_{j, \varepsilon, \kappa, K_{\mathcal{U}}}$ with probability as large as claimed, and by collecting all the error terms and by Remark 4.1, we see that for all $\varepsilon \in(0,1 / 10), j \geq j_{0}(\varepsilon), \kappa>0, f \in \mathcal{U}$ and $\omega \in$ $\Omega_{j, \varepsilon, \kappa, K_{\mathcal{U}}}$ (see Lemma 5.4),

$$
\begin{aligned}
\left\|\frac{f\left(\Lambda_{j}, \omega\right)}{\left|\Lambda_{j}\right|}-f^{*}\right\| & \leq\left(20 K_{f}+30 D_{f}\right) \varepsilon+\left(17 K_{f}+17 D_{f}+46\right) \beta(\varepsilon)+\kappa \\
& \leq\left(37 K_{f}+47 D_{f}+46\right) \sqrt{\varepsilon}+\kappa .
\end{aligned}
$$

Note the uniformity of the last inequality for all $f \in \mathcal{U}$ is also discussed in $\mathrm{Re}$ mark 4.1.

To see that the limit $f^{*}$ does not depend on the specific choice of $\left(\Lambda_{j}\right)$ use the following argument: Every two Følner sequences can be combined two one Følner sequence, which yields by our theory a limit $f^{*} \in \mathbb{B}$. As the two original sequences are subsequences, they lead to the same limit function $f^{*}$.

\section{APPENDIX A: CONDITIONAL RESAMPLING}

In Lemma 5.2, we need to remove the dependent parts of samples. We achieve this by resampling the critical parts of the samples, keeping the large enough already independent parts. This is done by augmenting the probability space to provide room for more random variables. The problem of resampling turned out to be treatable in a much broader setting, so a general tool is provided here.

THEOREM A.1 (Resampling). Let $(\Omega, \mathcal{A}, \mathbb{P})$ be a Borel probability space, $(S, \mathcal{S})$ a Borel space and $X: \Omega \rightarrow S$ an $S$-valued random variable with distribution $\mathbb{P}_{X}:=\mathbb{P} \circ X^{-1}: \mathcal{S} \rightarrow[0,1]$. Further, let $I$ be an index set, and for each $j \in I$, let $\mathcal{Y}_{j} \subseteq \mathcal{S}$ be a $\sigma$-algebra. 
Then there is a probability space $(\underline{\Omega}, \underline{\mathcal{A}}, \underline{\mathbb{P}})$, such that for all $j \in I$, maps as indicated in the following diagram exist and are measure preserving, and all the diagrams commute almost surely:

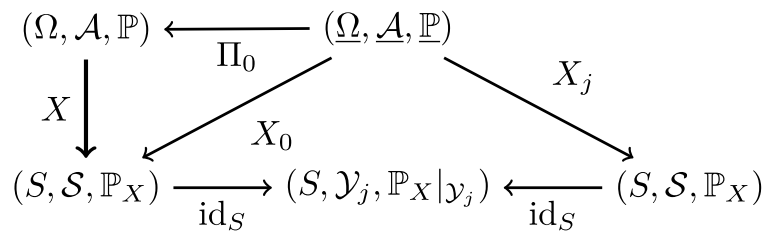

This means in particular that $\Pi_{0}$ is measure preserving, and that, for all $j \in I$ :

(i) the random variable $X_{j}$ has distribution $\mathbb{P}_{X}$,

(ii) for each measure space $(T, \mathcal{T})$ and each $\mathcal{Y}_{j}-\mathcal{T}$-measurable map $g:(S$, $\left.\mathcal{Y}_{j}\right) \rightarrow(T, \mathcal{T})$, we have $g\left(X_{0}\right)=g\left(X_{j}\right) \underline{\mathbb{P}}$-almost surely.

Furthermore, the joint distribution of $\left(X_{j}\right)_{j \in I}$ has the following properties:

(iii) For each finite subset $F \subseteq I$ and $A_{F}=\chi_{j \in F} A_{j}$, where $A_{j} \in \mathcal{S}$, we have $\mathbb{P}_{X}$-almost surely that

$$
\underline{\mathbb{P}}\left(X_{F} \in A_{F} \mid X_{0}=\cdot\right)=\prod_{j \in F} \underline{\mathbb{P}}\left(X_{j} \in A_{j} \mid X_{0}=\cdot\right)=\prod_{j \in F} \mathbb{P}_{X}\left(A_{j} \mid \mathcal{Y}_{j}\right)
$$

In particular, the random variables $X_{j}, j \in I$, are independent when conditioned on $X_{0}$.

(iv) If, for a (not necessarily finite) subset $J \subseteq I$, the $\sigma$-algebras $\mathcal{Y}_{j}, j \in J$, are $\mathbb{P}_{X}$-independent, then the random variables $X_{j}, j \in J$, are $\mathbb{P}$-independent.

Since $\Pi_{0}$ is measure preserving, $(\underline{\Omega}, \underline{\mathcal{A}}, \underline{\mathbb{P}})$ extends $(\Omega, \mathcal{A}, \mathbb{P})$. Property (i) justifies the name resampling. Statement (ii) says that in $X_{j}$ the information contained in $\mathcal{Y}_{j}$ is preserved throughout the resampling, $j \in I$. Point (iii) states that the new random variables copied only the information from $\mathcal{Y}_{j}, j \in I$, and not more. In (iv), we learn how to provide independence of the resampling random variables.

PROOF. We define the spaces and maps as follows:

$$
\begin{aligned}
& \underline{\Omega}:=\Omega \times S^{I}, \quad \underline{\mathcal{A}}:=\mathcal{A} \otimes \mathcal{S}^{\otimes I}, \\
& \Pi_{0}: \underline{\Omega} \rightarrow \Omega, \Pi_{0}\left(\omega,\left(s_{j}\right)_{j \in I}\right):=\omega, \\
& X_{0}: \underline{\Omega} \rightarrow S, X_{0}\left(\omega,\left(s_{j}\right)_{j \in I}\right):=X(\omega), \\
& X_{j}: \underline{\Omega} \rightarrow S, X_{j}\left(\omega,\left(s_{k}\right)_{k \in I}\right):=s_{j} .
\end{aligned}
$$

We now define the measure $\underline{\mathbb{P}}$ via Kolmogorov's extension theorem; see [4], Theorem 14.36. We need a consistent family of probability measures. For a more unifying notation, we augment $I_{0}:=\{0\} \dot{\cup} I$. Fix a finite subset $F \subseteq I_{0}$. If $0 \in F$, we 
define a probability measure $\mathbb{P}^{F}: \mathcal{A} \otimes \mathcal{S}^{\otimes F \backslash\{0\}} \rightarrow[0,1]$. In the case $0 \notin F$, we define a probability measure $\mathbb{P}^{F}: \mathcal{S}^{\otimes F} \rightarrow[0,1]$. If $0 \in F$, then choose $A_{0} \in \mathcal{A}$; otherwise, let $A_{0}:=\Omega$. For all $j \in F \backslash\{0\}$, we let $A_{j} \in \mathcal{S}$. Now let $A_{F}:=\times_{j \in F} A_{j}$ and

$$
\mathbb{P}^{F}\left(A_{F}\right):=\mathbb{E}\left[\mathbf{1}_{A_{0}} \prod_{j \in F \backslash\{0\}} \mathbb{P}_{X}\left(A_{j} \mid \mathcal{Y}_{j}\right) \circ X\right]
$$

Here, $\mathbb{E}$ denotes integration with respect to $\mathbb{P}$. By the extension theorem for measures (see [4], Theorem 1.53), (A.1) defines a probability measure. The family $\left(\mathbb{P}^{F}\right) F \subseteq I$ finite is consistent. For example, for finite subsets $0 \notin F \subseteq J \subseteq I$ with the projection $\Pi_{F}^{J}: S^{J} \rightarrow S^{F}$ and $A_{F}=\times_{j \in F} A_{j}$ with $A_{j} \in \mathcal{S}$, we have $\left(\Pi_{F}^{J}\right)^{-1}\left(A_{F}\right)=A_{F} \times \times_{j \in J \backslash F} S$. Thus,

$$
\mathbb{P}^{J}\left(\left(\Pi_{F}^{J}\right)^{-1}\left(A_{F}\right)\right)=\mathbb{E}_{X}\left[\prod_{j \in F} \mathbb{P}_{X}\left(A_{j} \mid \mathcal{Y}_{j}\right) \prod_{j \in J \backslash F} \mathbb{P}_{X}\left(S \mid \mathcal{Y}_{j}\right)\right]=\mathbb{P}^{F}\left(A_{F}\right),
$$

where $\mathbb{E}_{X}$ is integration with respect to $\mathbb{P}_{X}$. The remaining cases $0 \in F \subseteq J$, and $0 \notin F$ but $0 \in J$ work analogously. By Kolmogorov's extension theorem, we have exactly one measure $\underline{\mathbb{P}}:=\lim _{\leftarrow \subseteq I} \mathbb{P}^{F}: \underline{\mathcal{A}} \rightarrow[0,1]$.

We now verify the properties of $\mathbb{P}$. Let us first check that $\Pi_{0}$ is measure preserving. Indeed, for $A \in \mathcal{A}$, we have

$$
\underline{\mathbb{P}}\left(\Pi_{0} \in A\right)=\mathbb{P}^{\{0\}}(A)=\mathbb{E}\left[\mathbf{1}_{A}\right]=\mathbb{P}(A) .
$$

Now we already know that $X_{0}=X \circ \Pi_{0}$ is measure preserving, also.

Ad (i): For all $j \in I$ and $B \in \mathcal{S}$, we have

$$
\underline{\mathbb{P}}\left(X_{j} \in B\right)=\mathbb{P}^{\{j\}}(B)=\mathbb{E}_{X}\left[\mathbb{P}_{X}\left(B \mid \mathcal{Y}_{j}\right)\right]=\mathbb{E}_{X}\left[\mathbf{1}_{B}\right]=\mathbb{P}_{X}(B) .
$$

Ad (ii): Let $j \in I,(T, \mathcal{T})$ be a measure space and $g: S \rightarrow T$ be $\mathcal{Y}_{j}-\mathcal{T}$ measurable. We determine the joint distribution of $X$ and $X_{j}$. By (A.1), we have, for $B, B^{\prime} \in \mathcal{T}$, that $A:=g^{-1}(B) \in \mathcal{Y}_{j}$ as well as $A^{\prime}:=g^{-1}\left(B^{\prime}\right) \in \mathcal{Y}_{j}$, and

$$
\begin{aligned}
\mathbb{P}\left(g\left(X_{0}\right) \in B, g\left(X_{j}\right) \in B^{\prime}\right) & =\underline{\mathbb{P}}\left(X_{0} \in A, X_{j} \in A^{\prime}\right) \\
& =\mathbb{P}^{\{0, j\}}\left(X^{-1}(A) \times A^{\prime}\right) \\
& =\mathbb{E}\left[\mathbf{1}_{X^{-1}(A)} \mathbb{P}_{X}\left(A^{\prime} \mid \mathcal{Y}_{j}\right) \circ X\right] \\
& =\mathbb{E}_{X}\left[\mathbf{1}_{A} \mathbf{1}_{A^{\prime}}\right] \\
& =\mathbb{P}_{X}\left(A \cap A^{\prime}\right) \\
& =\mathbb{P}\left(X_{0} \in A \cap A^{\prime}\right) \\
& =\mathbb{P}\left(g\left(X_{0}\right) \in B \cap B^{\prime}\right),
\end{aligned}
$$


where in the last line, we used that $A \cap A^{\prime}=g^{-1}(B) \cap g^{-1}\left(B^{\prime}\right)=g^{-1}(B \cap$ $\left.B^{\prime}\right)$. Now, since the rectangles $\left\{B \times B^{\prime} \mid B, B^{\prime} \in \mathcal{T}\right\}$ are stable under intersections and generate $\mathcal{T} \otimes \mathcal{T}$, equation (A.2) determines the distribution of $\left(g\left(X_{0}\right), g\left(X_{j}\right)\right): \underline{\Omega} \rightarrow T^{2}$. Note that the measure which is concentrated on the diagonal $\{(t, t) \mid t \in T\}$ with both marginals equal to $\mathbb{P}_{X} \circ g^{-1}$ satisfies (A.2), also. Therefore, $\mathbb{P}\left(g\left(X_{0}\right)=g\left(X_{j}\right)\right)=1$.

Ad (iii): Fix a finite subset $F \subseteq I$ and $A_{j} \in \mathcal{S}$ for $j \in F$, and let $A_{F}:=$ $\times_{j \in F} A_{j}$. For all $B \in \mathcal{S}$, we have

$$
\begin{aligned}
\underline{\mathbb{E}}\left[\mathbf{1}_{\left\{X_{0} \in B\right\}} \mathbb{P}\left(X_{F} \in A_{F} \mid X_{0}\right)\right] & =\underline{\mathbb{E}}\left[\mathbf{1}_{\left\{X_{0} \in B\right\}} \underline{\mathbb{E}}\left[\mathbf{1}_{\left\{X_{F} \in A_{F}\right\}} \mid X_{0}\right]\right] \\
& =\underline{\mathbb{E}}\left[\mathbf{1}_{\left\{X_{0} \in B\right\}} \mathbf{1}_{\left\{X_{F} \in A_{F}\right\}}\right] \\
& =\underline{\mathbb{P}}\left[X_{0} \in B, X_{F} \in A_{F}\right] \\
& =\mathbb{P}\{0\} \cup F\left(X^{-1}(B) \times A_{F}\right) \\
& =\mathbb{E}\left[\mathbf{1}_{X^{-1}(B)} \prod_{j \in F} \mathbb{P}_{X}\left(A_{F} \mid \mathcal{Y}_{j}\right) \circ X\right] \\
& =\underline{\mathbb{E}}\left[\mathbf{1}_{\left\{X_{0} \in B\right\}} \prod_{j \in F} \mathbb{P}_{X}\left(A_{F} \mid \mathcal{Y}_{j}\right) \circ X_{0}\right] .
\end{aligned}
$$

Since $\sigma\left(X_{0}\right)=\left\{\left\{X_{0} \in B\right\} \mid B \in \mathcal{S}\right\}$, this proves

$$
\underline{\mathbb{P}}\left(X_{F} \in A_{F} \mid X_{0}\right)=\prod_{j \in F} \mathbb{P}_{X}\left(X_{j} \in A_{j} \mid \mathcal{Y}_{j}\right) \circ X_{0}
$$

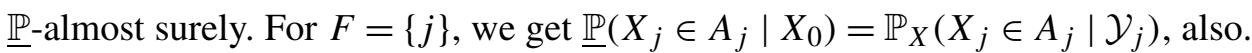
The claim is the factorized version of these statements, which exist because $(S, \mathcal{S})$ is a Borel space.

Ad (iv): For $F \subseteq J$ finite and $A_{F}=\times_{j \in F} A_{j}$ with $A_{j} \in \mathcal{S}$, we use (iii) to get

$$
\begin{aligned}
\mathbb{P}\left(X_{F} \in A_{F}\right) & =\underline{\mathbb{E}}\left[\underline{\mathbb{P}}\left(X_{F} \in A_{F} \mid X_{0}\right)\right] \\
& =\underline{\mathbb{E}}\left[\prod_{j \in F} \mathbb{P}_{X}\left(A_{j} \mid \mathcal{Y}_{j}\right) \circ X_{0}\right] \\
& =\mathbb{E}_{X}\left[\prod_{j \in F} \mathbb{P}_{X}\left(A_{j} \mid \mathcal{Y}_{j}\right)\right] .
\end{aligned}
$$

The $\sigma$-algebras $\mathcal{Y}_{j}, j \in F \subseteq J$, are $\mathbb{P}_{X}$-independent. This independence is inherited by $\mathcal{Y}_{j}$-measurable functions like $\mathbb{P}_{X}\left(A_{j} \mid \mathcal{Y}_{j}\right)$. We can therefore continue the calculation with

$$
\underline{\mathbb{P}}\left(X_{F} \in A_{F}\right)=\prod_{j \in F} \mathbb{E}_{X}\left[\mathbb{P}_{X}\left(A_{j} \mid \mathcal{Y}_{j}\right)\right]=\prod_{j \in F} \mathbb{P}_{X}\left(A_{j}\right)=\prod_{j \in F} \mathbb{P}\left(X_{j} \in A_{j}\right) .
$$

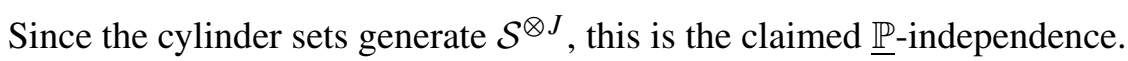




\section{APPENDIX B: PROOF SUMMARY FOR MONTILABLE AMENABLE GROUPS}

The proofs of [12] concerning the case $G=\mathbb{Z}^{d}$ can be generalized to apply to a finitely generated amenable group $G$ if it satisfies the tiling property $(\boxplus)$.

We list the major changes which are necessary for this purpose:

(a) Instead of defining the set $T_{m, n}$ using multiples of $m$ (cf. equation (4.1) in [12]), we employ the grid $T_{m}$, namely, we set

$$
T_{m, n}:=\left\{t \in T_{m} \mid \Lambda_{m} t \subseteq \Lambda_{n}\right\}
$$

Thus, $T_{m, n}$ contains the elements of $T_{m}$ which correspond to translates of $\Lambda_{m}$ which are completely contained in $\Lambda_{n}$. Using this definition, the empirical measures are $L_{m, n}^{\omega}$ and $L_{m, n}^{\omega, r}$ are given accordingly.

(b) One needs to verify the following basic result. Given a tiling Følner sequence $\left(\Lambda_{n}\right)$, we have:

(i) for each $m \in \mathbb{N}$, the sequence $\left(\Lambda_{m} T_{m, n}\right)_{n \in \mathbb{N}}$ is a Følner sequence;

(ii) for each $m, n \in \mathbb{N}$, we have $\Lambda_{n} \subseteq \partial^{\rho(m)}\left(\Lambda_{n}\right) \cup \Lambda_{m} T_{m, n}$, where $\rho(m)=$ $\operatorname{diam}\left(\Lambda_{m}\right)$; and

(iii) for each $m \in \mathbb{N}$ we have $\lim _{n \rightarrow \infty}\left|\Lambda_{n}\right| /\left|T_{m, n}\right|=\left|\Lambda_{m}\right|$.

(c) Points (a) and (b) allow to prove an equivalent version of Lemma 4.1 of [12] in the situation of amenable groups with property $(\boxplus)$, by following exactly the steps of the proof presented therein.

(d) Besides Lemma 4.1 in [12], also Lemma 6.1 in [12] needs to be slightly changed. In fact, again by using (a) and (b) the proof can directly be adapted to the situation where $G$ is amenable and $\left(\Lambda_{n}\right)$ is a tiling Følner sequence.

(e) In the end, the proof of the main theorem reduces basically to an application of the triangle inequality, the new versions of Lemma 4.1 and Lemma 6.1 as well as Theorem 5.6 in [12]. Note that Theorem 5.6 need not to be adapted as it is independent of the geometry.

\section{REFERENCES}

[1] AdAchi, T. (1993). A note on the Følner condition for amenability. Nagoya Math. J. 131 67-74. MR1238633

[2] Aliprantis, C. D. and Border, K. C. (2006). Infinite Dimensional Analysis: A Hitchhiker's Guide, 3rd ed. Springer, Berlin. MR2378491

[3] Dehardt, J. (1971). Generalizations of the Glivenko-Cantelli theorem. Ann. Math. Stat. 42 2050-2055.

[4] Klenke, A. (2008). Probability Theory. Number 223 in Universitext, 1 st ed. Springer, London.

[5] Krieger, F. (2009). Le lemme d'Ornstein-Weiss d'aprés Gromov. Dyn., Ergod. Theory Geom. 54 99-112.

[6] LenZ, D., Müller, P. and Veselić, I. (2008). Uniform existence of the integrated density of states for models on $\mathbb{Z}^{d}$. Positivity 12 571-589. MR2448749 
[7] Lenz, D., Schwarzenberger, F. and Veselić, I. (2010). A Banach space-valued ergodic theorem and the uniform approximation of the integrated density of states. Geom. Dedicata 150 1-34.

[8] Lenz, D. and Stollmann, P. (2005). An ergodic theorem for Delone dynamical systems and existence of the integrated density of states. J. Anal. Math. 97 1-24. MR2274971

[9] LENZ, D. and VEsELIĆ, I. (2009). Hamiltonians on discrete structures: Jumps of the integrated density of states and uniform convergence. Math. Z. 263 813-835.

[10] ORNSTEIN, D. and Weiss, B. (1987). Entropy and isomorphism theorems for actions of amenable groups. J. Anal. Math. 48 1-141.

[11] Pogorzelski, F. and Schwarzenberger, F. (2016). A Banach space-valued ergodic theorem for amenable groups and applications. J. Anal. Math. 130 19-69.

[12] Schumacher, C., Schwarzenberger, F. and Veselić, I. (2017). A Glivenko-Cantelli theorem for almost additive functions on lattices. Stochastic Process. Appl. 127 179-208.

[13] SchwarZenberger, F. (2013). The integrated density of states for operators on groups. Ph.D. thesis, Technische Universität Chemnitz. Available at http://nbn-resolving.de/urn: nbn:de:bsz:ch1-qucosa-123241.

[14] TAO, T. (2015). Failure of the $L^{1}$ pointwise and maximal ergodic theorems for the free group. Forum Math. Sigma 319.

[15] Veselić, I. (2005). Spectral analysis of percolation Hamiltonians. Math. Ann. 331 841-865.

[16] Weiss, B. (2001). Monotileable amenable groups. In Topology, Ergodic Theory, Real Algebraic Geometry. Amer. Math. Soc. Transl. Ser. 202 257-262. Amer. Math. Soc., Providence, RI. MR1819193

[17] Wright, F. T. (1981). The empirical discrepancy over lower layers and a related law of large numbers. Ann. Probab. 9 323-329. MR0606996

C. SCHUMACHER

I. VESELIĆ

FAKULTÄT FÜR MATHEMATIK

TU DORTMUND

44221 DORTMUND

GERMANY

E-MAIL: christoph.schumacher@math.tu-dortmund.de ivan.veselic@math.tu-dortmund.de
F. SCHWARZENBERGER

FAKULTÄT FÜR INFORMATIK/MATHEMATIK

HTW DRESDEN

01069 DRESDEN

GERMANY

E-MAIL: fabian.schwarzenberger@htw-dresden.de 